Homology, Homotopy and Applications, vol.9(2), 2007, pp.413-444

\title{
COFIBRATIONS IN THE CATEGORY OF FRÖLICHER SPACES: PART I
}

\author{
BRETT DUGMORE AND PATRICE PUNGU NTUMBA
}

\author{
(communicated by Ronald Brown)
}

\begin{abstract}
Cofibrations are defined in the category of Frölicher spaces by weakening the analog of the classical definition to enable smooth homotopy extensions to be more easily constructed, using flattened unit intervals. We later relate smooth cofibrations to smooth neighborhood deformation retracts. The notion of smooth neighborhood deformation retract gives rise to an analogous result that a closed Frölicher subspace $A$ of the Frölicher space $X$ is a smooth neighborhood deformation retract of $X$ if and only if the inclusion $i: A \hookrightarrow X$ comes from a certain subclass of cofibrations. As an application we construct the right Puppe sequence.
\end{abstract}

\section{Preliminaries}

The purpose of this section is to briefly survey the notion of Frölicher spaces, and to present our approach to homotopy theory in the category of Frölicher spaces. Frölicher spaces arise naturally in physics, and generalize the concept of smooth manifolds. A Frölicher space, or smooth space as initially called by Frölicher and Kriegl [8], is a triple $\left(X, \mathcal{C}_{X}, \mathcal{F}_{X}\right)$ consisting of a set $X$, and subsets $\mathcal{C}_{X} \subseteq X^{\mathbb{R}}$, $\mathcal{F}_{X} \subseteq \mathbb{R}^{X}$ such that

- $\mathcal{F}_{X} \circ \mathcal{C}_{X}=\left\{f \circ c \mid f \in \mathcal{F}_{X}, c \in \mathcal{C}_{X}\right\} \subseteq C^{\infty}(\mathbb{R})$

- $\Phi \mathcal{C}_{X}:=\left\{f: X \rightarrow \mathbb{R} \mid f \circ c \in C^{\infty}(\mathbb{R})\right.$ for all $\left.c \in \mathcal{C}_{X}\right\}=\mathcal{F}_{X}$

- $\Gamma \mathcal{F}_{X}:=\left\{c: \mathbb{R} \rightarrow X \mid f \circ c \in C^{\infty}(\mathbb{R})\right.$ for all $\left.f \in \mathcal{F}_{X}\right\}=\mathcal{C}_{X}$.

Frölicher and Kriegl [8], and Kriegl and Michor [11] are our main references for Frölicher spaces. The following terminology will be used in the paper: Given a Frölicher space $\left(X, \mathcal{C}_{X}, \mathcal{F}_{X}\right)$, the pair $\left(\mathcal{C}_{X}, \mathcal{F}_{X}\right)$ is called a smooth structure; the elements of $\mathcal{C}_{X}$ and $\mathcal{F}_{X}$ are called smooth curves and smooth functions respectively. The topology assumed for a Frölicher space $\left(X, \mathcal{C}_{X}, \mathcal{F}_{X}\right)$ throughout the paper is the initial topology $\mathcal{T}_{\mathcal{F}}$ induced by the set $\mathcal{F}_{X}$ of functions. When there is no fear of confusion, a Frölicher space $\left(X, \mathcal{C}_{X}, \mathcal{F}_{X}\right)$ will simply be denoted $X$. The most natural Frölicher spaces are the finite-dimensional smooth manifolds, where if $X$ is

Received May 7, 2007, revised September 3, 2007; published on November 8, 2007. 2000 Mathematics Subject Classification: 55P05.

Key words and phrases: Frölicher space, flattened unit interval, smooth neighborhood deformation retract, smooth cofibration, cofibration with FCIP, Puppe sequence.

Copyright (C) 2007, International Press. Permission to copy for private use granted. 
such a smooth manifold, then $\mathcal{C}_{X}$ and $\mathcal{F}_{X}$ consist of all smooth curves $\mathbb{R} \rightarrow X$ and smooth functions $X \rightarrow \mathbb{R}$. Euclidean finite-dimensional smooth manifolds $\mathbb{R}^{n}$, when viewed as Frölicher spaces, are called Euclidean-Frölicher spaces. In the sequel, by $\mathbb{R}^{n}, n \in \mathbb{N}$, we mean the Frölicher space $\mathbb{R}^{n}$, equipped with its usual smooth manifold structure.

A Frölicher space $X$ is called Hausdorff if and only if the smooth real-valued functions on $X$ are point-separating, i.e. if and only if $\mathcal{T}_{\mathcal{F}}$ is Hausdorff.

A Frölicher structure $\left(\mathcal{C}_{X}, \mathcal{F}_{X}\right)$ on a set $X$ is said to be generated by a set $F_{0} \subseteq \mathbb{R}^{X}$ (resp. $C_{0} \subseteq X^{\mathbb{R}}$ ) if $\mathcal{C}_{X}=\Gamma F_{0}$ and $\mathcal{F}_{X}=\Phi \Gamma F_{0}$ (resp. $\mathcal{F}_{X}=\Phi C_{0}$ and $\mathcal{C}_{X}=$ $\left.\Gamma \Phi C_{0}\right)$. Note that different sets $F_{0} \subseteq \mathbb{R}^{X}$ on the same set $X$ may give rise to a same smooth structure on $X$. A set mapping $\varphi: X \rightarrow Y$ between Frölicher spaces is called a map of Frölicher spaces or just a smooth map if for each $f \in \mathcal{F}_{Y}$, the pullback $f \circ \varphi \in \mathcal{F}_{X}$. This is equivalent to saying that for each $c \in \mathcal{C}_{X}, \varphi \circ c \in \mathcal{C}_{Y}$. An injective smooth map $\varphi: X \rightarrow Y$ is called an embedding if the smooth structure on $X$ is the initial structure corresponding to $\varphi$; i.e. $\mathcal{C}_{X}=\Gamma\left(\mathcal{F}_{Y} \circ \varphi\right)$. The following result is relevant.

Lemma 1.1. Let $f: X \rightarrow Y$, and $g: Y \rightarrow Z$ be maps of Frölicher spaces. Suppose that $f$ is injective; therefore, if $g \circ f: X \rightarrow Z$ is an embedding, then $f$ is an embedding too.

Some useful facts regarding Frölicher spaces can be gathered in the following

Theorem 1.2. The category $\mathbb{F} \mathbb{R} \mathbb{L}$ is complete (i.e. arbitrary limits exist), cocomplete (i.e. arbitrary colimits exist), and Cartesian closed.

Given a collection of Frölicher spaces $\left\{X_{i}\right\}_{i \in I}$, let $X=\prod_{i \in I} X_{i}$ be the set product of the sets $\left\{X_{i}\right\}_{i \in I}$ and $\pi_{i}: X \rightarrow X_{i}, i \in I$ denote the projection map $\left(x_{i}\right)_{i \in I} \mapsto x_{i}$. The initial structure on $X$ is generated by the set

$$
F_{0}=\bigcup_{i \in I}\left\{f \circ \pi_{i}: f \in \mathcal{F}_{X_{i}}\right\} .
$$

The ensuing Frölicher space $\left(X, \Gamma F_{0}, \varphi \Gamma F_{0}\right)$ is called the product space of the family $\left\{X_{i}\right\}_{i \in I}$. Clearly,

$$
\Gamma F_{0}=\left\{c: \mathbb{R} \rightarrow X \mid \text { if } c(t)=\left(c_{i}(t)\right)_{i \in I}, \text { then } c_{i} \in \mathcal{C}_{X_{i}} \text { for every } i \in I\right\} .
$$

Now, let $\biguplus_{i \in I} X_{i}$ be the disjoint union of sets $\left\{X_{i}\right\}_{i \in I}$, and $\iota_{X_{i}}: X_{i} \rightarrow \biguplus_{i \in I} X_{i}$ the inclusion map. Place the smooth final structure on $\biguplus_{i \in I} X_{i}$ corresponding to the family $\left\{\iota_{X_{i}}\right\}_{i \in I}$. The resulting Frölicher space is called the coproduct of $\left\{X_{i}\right\}_{i \in I}$, denoted $\coprod_{i \in I} X_{i}$, and

$$
\mathcal{F}_{\bigsqcup_{i \in I} X_{i}}=\left\{f: \coprod_{i \in I} X_{i} \rightarrow \mathbb{R} \mid \text { for each } i \in I,\left.f\right|_{X_{i}} \in \mathcal{F}_{X_{i}}\right\}
$$

is the collection of smooth functions for the coproduct.

Corollary 1.3. Let $X, Y$, and $Z$ be Frölicher spaces. Then the following canonical mappings are smooth.

- $e v: C^{\infty}(X, Y) \times X \rightarrow Y,(f, x) \mapsto f(x)$ 
- ins: $X \rightarrow C^{\infty}(Y, X \times Y), x \mapsto(y \mapsto$ ins $(x)(y)=(x, y))$

- comp: $C^{\infty}(Y, Z) \times C^{\infty}(X, Y) \rightarrow C^{\infty}(X, Z),(g, f) \mapsto g \circ f$

- $f_{*}: C^{\infty}(X, Y) \rightarrow C^{\infty}(X, Z), f_{*}(g)=f \circ g$, where $f \in C^{\infty}(Y, Z)$

- $g_{*}: C^{\infty}(Z, Y) \rightarrow C^{\infty}(X, Y), g_{*}(f)=f \circ g$, where $g \in C^{\infty}(X, Z)$.

Corollary 1.4. Let $X, Y$, and $Z$ be Frölicher spaces. The mapping $\psi:(X \times Y) \times$ $Z \rightarrow X \times(Y \times Z),((x, y), z) \mapsto(x,(y, z))$, is an isomorphism.

Because of this isomorphism, the spaces $(X \times Y) \times Z$ and $X \times(Y \times Z)$ are denoted $X \times Y \times Z$.

Given Frölicher spaces $X, Y$, and $Z$ and in view of the cartesian closedness of the category $\mathbb{F R L}$, the exponential law

$$
C^{\infty}(X \times Y, Z) \cong C^{\infty}\left(X, C^{\infty}(Y, Z)\right)
$$

holds. Because $\mathcal{F}_{X}=C^{\infty}(X, \mathbb{R})$, it follows by cartesian closedness of $\mathbb{F} \mathbb{R} \mathbb{L}$ that the collection $\mathcal{F}_{X}$ can be made into a Frölicher space on its own right.

Finally we would like to show how to construct smooth braking functions, following Hirsch [9]. Smooth braking functions are tools that are behind most results in this paper. In $[\mathbf{1 2}]$, it is shown that the function $\varphi: \mathbb{R} \rightarrow \mathbb{R}$ given by

$$
\varphi(u)= \begin{cases}0 & \text { if } u \leqslant 0 \\ e^{\frac{-1}{u}} & \text { if } u>0\end{cases}
$$

is smooth. Substituting $x^{2}$ for $u$ in the above function, one sees that the function $\psi: \mathbb{R} \rightarrow \mathbb{R}$, given by

$$
\psi(x)= \begin{cases}0 & \text { if } x \leqslant 0 \\ e^{\frac{-1}{x^{2}}} & \text { if } u>0\end{cases}
$$

is smooth. Now, let us construct a smooth function $\alpha: \mathbb{R} \rightarrow \mathbb{R}$ with the following properties. Let $0 \leqslant a<b . \alpha(t)$ should satisfy:

- $\alpha(t)=0$ for $t \leqslant a$

- $0<\alpha(t)<1$ for $a<t<b$

- $\alpha$ is strictly increasing for $a<t<b$

- $\alpha(t)=1$ for $t \geqslant b$.

Define $\alpha: \mathbb{R} \rightarrow[0,1]$ by

$$
\alpha(t)=\frac{\int_{a}^{t} \gamma(x) d x}{\int_{a}^{b} \gamma(x) d x},
$$

where $\gamma(x)=\psi(x-a) \psi(b-x)$.

In the sequel, the notation $\alpha_{\epsilon}, 0<\epsilon<\frac{1}{2}$, will refer to a smooth braking function with the following properties:

- $\alpha_{\epsilon}(t)=0$ for $t \leqslant \epsilon$

- $0<\alpha_{\epsilon}(t)<1$ for $\epsilon<t<1-\epsilon$

- $\alpha$ strictly increasing for $\epsilon<t<1-\epsilon$

- $\alpha_{\epsilon}(t)=1$ for $1-\epsilon \leqslant t$. 
One might begin investigating homotopy theory in $\mathbb{F} \mathbb{R} \mathbb{L}$ by simply following the homotopy theory of topological spaces, replacing continuous functions with smooth ones. One can certainly define the notion of a homotopy $H: I \times X \rightarrow Y$ between smooth maps $H(0,-)$ and $H(1,-)$ in this way. One can even get as far as the left Puppe sequence (see [5]), but eventually difficulties begin to arise.

Extending functions defined on a subspace of a Frölicher space tends to be a little tricky, and so the definition of a cofibration in $\mathbb{F} \mathbb{R L}$ is one that needs careful consideration. To do this we define a slightly weaker notion of cofibration than the notion obtained from topological spaces. In addition, we define the mapping cylinder of a smooth map $f: X \rightarrow Y$ using not the unit interval, but a modified version called the weakly flattened unit interval, denoted $\mathbb{I}$, which, as one can show, is topologically homeomorphic to the unit interval. This modified structure on the unit interval allows us to show that the inclusion of a space $X$ into the mapping cylinder of $f: X \rightarrow Y$ is a cofibration (in our weaker sense).

The weakly flattened unit interval is useful, but it also has its drawbacks. It would be ideal to have a single structure on the unit interval that can be used throughout our homotopy theory, but the weakly flattened unit interval is not suitable because it has the rather restrictive property that a smooth map $f: I \rightarrow I$ on the usual unit interval often does not define a smooth map $f: \mathbb{I} \rightarrow \mathbb{I}$, unless the endpoints of the interval are mapped to the endpoints. This restrictive property means that we only use the flattened unit intervals where they are absolutely necessary.

\section{Basic constructions of homotopy theory in $\mathbb{F} \mathbb{R} L$}

In this section, we define the fundamental notions of homotopy theory in the category $\mathbb{F} \mathbb{R} \mathbb{L}$, such as the homotopy relation and the mapping cylinder.

\subsection{Flattened structures on the unit interval}

We define two main Frölicher structures which we call the flattened unit interval and the weakly flattened unit interval. Let $\left(\mathcal{C}_{I}, \mathcal{F}_{I}\right)$ be the subspace structure induced on $I$ by the inclusion $I \hookrightarrow \mathbb{R}$.

Definition 2.1. The Frölicher space $\left(\mathbf{I}, \mathcal{C}_{\mathbf{I}}, \mathcal{F}_{\mathbf{I}}\right)$, where the structure $\left(\mathcal{C}_{\mathbf{I}}, \mathcal{F}_{\mathbf{I}}\right)$ is the structure generated by the set

$$
\begin{aligned}
& F=\left\{f \in \mathcal{F}_{I} \mid \text { there exists } 0<\epsilon<\frac{1}{4} \text { with } f(t)=f(0) \quad \text { for } t \in[0, \epsilon)\right. \text { and } \\
& f(t)=f(1) \text { for } t \in(1-\epsilon, 1]\},
\end{aligned}
$$

is called the flattened unit interval.

It is easy to see that any continuous map $c: \mathbb{R} \rightarrow[0,1]$ defines a structure curve on $\mathbf{I}$ if and only if it is smooth at every point $t \in \mathbb{R}$, where $c(t) \in(0,1)$.

We define the left (resp. right) flattened unit interval, denoted by $\mathbf{I}^{-}$(resp. $\mathbf{I}^{+}$), to be the Frölicher space whose underlying set is the unit interval $[0,1]$, and structure is the structure generated by the structure functions in $\mathcal{F}_{I}$ that are constant near 0 (resp. 1). 
Definition 2.2. The Frölicher space $\left(\mathbb{I}, \mathcal{C}_{\mathbb{I}}, \mathcal{F}_{\mathbb{I}}\right)$, with the structure defined below, is called the weakly flattened unit interval. The underlying set is the unit interval; the structure $\left(\mathcal{C}_{\mathbb{I}}, \mathcal{F}_{\mathbb{I}}\right)$ is generated by the family

$$
F=\left\{f \in \mathcal{F}_{I} \mid \lim _{t \rightarrow 0^{+}} \frac{d^{n}}{d t^{n}} f(t)=0, \lim _{t \rightarrow 1^{-}} \frac{d^{n}}{d t^{n}} f(t)=0, n \geqslant 1\right\} .
$$

We call the property, for all $f \in F$,

$$
\lim _{t \rightarrow 0^{+}} \frac{d^{n}}{d t^{n}} f(t)=0, \lim _{t \rightarrow 1^{-}} \frac{d^{n}}{d t^{n}} f(t)=0, n \geqslant 1,
$$

the zero derivative property of $f$.

We shall prove that all structure functions on II have the zero derivative property; in other words, $\mathcal{F}_{\mathbb{I}}=F$. To that effect, we need the following lemma.

Lemma 2.3. Let $c: \mathbb{R} \rightarrow \mathbb{R}$ be a smooth real-valued function at $t=t_{0}$, and let $f: \mathbb{R} \rightarrow \mathbb{R}$ be a smooth real-valued function at $t=c\left(t_{0}\right)$. Then,

$$
\begin{aligned}
& \frac{d^{n}}{d t^{n}}(f \circ c)\left(t_{0}\right)=f^{(n)}\left(c\left(t_{0}\right)\right)\left(c^{\prime}\left(t_{0}\right)\right)^{n}+\text { terms of the form } \\
& \qquad a f^{(k)}\left(c\left(t_{0}\right)\right)\left(c^{\prime}\left(t_{0}\right)\right)^{m_{1}}\left(c^{\prime \prime}\left(t_{0}\right)\right)^{m_{2}} \ldots\left(c^{(n-1)}\left(t_{0}\right)\right)^{m_{n-1}},
\end{aligned}
$$

where $k<n$ and $a \in \mathbb{R}$. In addition, if $a \neq 0$, then at least one of $m_{2}, m_{3}, \ldots, m_{n-1}$ is also non-zero.

Proof. The proof is done by induction. For the sake of brevity, we call the term $f^{(n)}\left(c\left(t_{0}\right)\right)\left(c^{\prime}\left(t_{0}\right)\right)^{n}$ the primary term for $n$, and the terms of the form

$$
a f^{(k)}\left(c\left(t_{0}\right)\right)\left(c^{\prime}\left(t_{0}\right)\right)^{m_{1}}\left(c^{\prime \prime}\left(t_{0}\right)\right)^{m_{2}} \ldots\left(c^{(n-1)}\left(t_{0}\right)\right)^{m_{n-1}}
$$

the lower order terms for $n$. The statement is true for $n=1$ and for $n=2$. Suppose the result is true for $n=k$. To show that the result holds for $n=k+1$, since

$$
\begin{aligned}
& \frac{d^{k+1}}{d t^{k+1}}(f \circ c)\left(t_{0}\right)=\frac{d}{d t}\left(f^{(k)}\left(c\left(t_{0}\right)\right)\left(c^{\prime}\left(t_{0}\right)\right)^{k}\right)+\text { terms of the form } \\
& \qquad \frac{d}{d t}\left(a f^{(j)}\left(c\left(t_{0}\right)\right)\left(c^{\prime}\left(t_{0}\right)\right)^{m_{1}}\left(c^{\prime \prime}\left(t_{0}\right)\right)^{m_{2}} \ldots\left(c^{(k-1)}\left(t_{0}\right)\right)^{m_{k-1}}\right),
\end{aligned}
$$

where $j<k+1$ and $a \in \mathbb{R}$, we need only show that

$$
\frac{d}{d t}\left(a f^{(j)}\left(c\left(t_{0}\right)\right)\left(c^{\prime}\left(t_{0}\right)\right)^{m_{1}}\left(c^{\prime \prime}\left(t_{0}\right)\right)^{m_{2}} \ldots\left(c^{(k-1)}\left(t_{0}\right)\right)^{m_{k-1}}\right)
$$

gives rise to lower terms for $n=k+1$, which is, by the way, straightforward.

Theorem 2.4. Let $F:=\mathcal{F}_{\mathbb{I}}=\left\{f \in \mathcal{F}_{I} \mid \lim _{t \rightarrow 0^{+}} \frac{d^{n}}{d t^{n}} f(t)=0=\lim _{t \rightarrow 1^{-}} \frac{d^{n}}{d t^{n}} f(t)\right\}$.

Proof. It is evident that $F \subseteq \mathcal{F}_{\mathbb{I}}$. We must show the reverse inequality. Let $0<\epsilon$ $<\frac{1}{2}$, and $0<M<1$. Consider the function $c_{M}: \mathbb{R} \rightarrow \mathbb{R}$, given by

$$
c_{M}(t)=\left(1-\alpha_{\epsilon}(|t|)\right) \beta_{M}(t)+\alpha_{\epsilon}(|t|),
$$

where $\alpha_{\epsilon}: \mathbb{R} \rightarrow \mathbb{R}$ is a smooth braking function as defined in the Preliminaries, and 
$\beta_{M}: \mathbb{R} \rightarrow \mathbb{R}$ is given by

$$
\beta_{M}(t)= \begin{cases}-M t & \text { if } t \leqslant 0 \\ t & \text { if } t>0\end{cases}
$$

It is easily seen that $c_{M}$ is continuous over all $\mathbb{R}$, and smooth over all $\mathbb{R}$ except at $t=0$. Also note that $0<c_{M}(t)<1$ for all $t \in \mathbb{R}$, and $c_{M}(t)=\beta_{M}(t)=0$ for all $0 \leqslant t<\epsilon$. Now,

$$
\begin{array}{llrl}
\frac{d}{d t} c_{M}(t) & =\frac{d}{d t} \beta_{M}(t)=-M, & & \text { for }-\epsilon<t<0 \\
\frac{d}{d t} c_{M}(t) & =\frac{d}{d t} \beta_{M}(t)=1, & & \text { for } 0<t<\epsilon .
\end{array}
$$

For $n>1$, we have

$$
\frac{d^{n}}{d t^{n}} c_{M}(t)=\frac{d^{n}}{d t^{n}} \beta_{M}(t)=0, \quad \text { for } t \in(-\epsilon, 0) \cup(0, \epsilon) .
$$

We now show that $c_{M} \in \Gamma F$. To this end, let $f \in F$. To show that $f \circ c_{M}: \mathbb{R} \rightarrow \mathbb{R}$ is smooth, it is obvious that we need only concentrate on the point $t=0$, because $f \circ c$ is smooth at every $t \neq 0$. It follows for $t \neq 0$, and $n \in \mathbb{N}$ that Lemma 2.3 applies. But as $t \rightarrow 0, c_{M}(t) \rightarrow 0^{+}$, and so, letting $s=c_{M}(t)$, we have

$$
\lim _{t \rightarrow 0} f^{(j)}\left(c_{M}(t)\right)=\lim _{s \rightarrow 0^{+}} f^{(j)}(s)=0,
$$

for all $j \in \mathbb{N}$, by the zero derivative property of $f$. Thus, as $t$ approaches the value 0 , the primary term and all the lower order terms of $\frac{d^{n}}{d t^{n}}\left(f \circ c_{M}\right)(t)$ vanish, and we have shown that $f \circ c_{M}$ is smooth at $t=0$. This implies that $f \circ c_{M} \in C^{\infty}(\mathbb{R}, \mathbb{R})$ for all $f \in F$. It follows that $c_{M} \in \Gamma F$.

We are now ready to show that $\mathcal{F}_{\mathbb{I}} \subseteq F$. To this end, suppose that we are given a structure function $f \in \mathcal{F}_{\mathbb{I}}$. We shall show that this $f$ has the zero derivative property, and is thus an element of $F$.

Since $f \in \mathcal{F}_{\mathbb{I}}$, we know that $f \circ c$ is a smooth real-valued function for every $c \in$ $\Gamma F$. In particular, $f \circ c_{M}$ is smooth for all $0<M<1$. Thus, for any $n \in \mathbb{N}$,

$$
\lim _{t \rightarrow 0^{-}} \frac{d^{n}}{d t^{n}}\left(f \circ c_{M}\right)(t)=\lim _{t \rightarrow 0^{+}} \frac{d^{n}}{d t^{n}}\left(f \circ c_{M}\right)(t) .
$$

As $t \rightarrow 0^{-}, c_{M}(t) \rightarrow 0^{+}$; let us consider the lower order terms for $n$. Each term of the form

$$
a f^{(k)}\left(c_{M}(t)\right)\left(c_{M}^{\prime}(t)\right)^{m_{1}}\left(c_{M}^{\prime \prime}(t)\right)^{m_{2}} \ldots\left(c_{M}^{(n-1)}(t)\right)^{m_{n-1}}
$$

has some term $\left(c_{M}^{(i)}(t)\right)^{m_{i}}$, for some $i>1$, with $m_{i} \neq 0$. But $\lim _{t \rightarrow 0^{-}} c_{M}^{(i)}(t)=0$, if $i>1$, and so

$$
\lim _{t \rightarrow 0^{-}} a f^{(k)}\left(c_{M}(t)\right)\left(c_{M}^{\prime}(t)\right)^{m_{1}}\left(c_{M}^{\prime \prime}(t)\right)^{m_{2}} \ldots\left(c_{M}^{(n-1)}(t)\right)^{m_{n-1}}=0
$$


So all the lower order terms fall away; therefore

$$
\begin{aligned}
\lim _{t \rightarrow 0^{-}} \frac{d^{n}}{d t^{n}}\left(f \circ c_{M}\right)(t) & =\lim _{t \rightarrow 0^{-}} f^{(n)}\left(c_{M}(t)\right)\left(c_{M}^{\prime}(t)\right)^{n} \\
& =\lim _{t \rightarrow 0^{-}} f^{(n)}\left(c_{M}(t)\right)(-M)^{n} \\
& =\lim _{s \rightarrow 0^{+}} f^{(n)}(s)(-M)^{n}
\end{aligned}
$$

where $s=c_{M}(t)$. In a similar way one shows that

$$
\lim _{t \rightarrow 0^{+}} \frac{d^{n}}{d t^{n}}\left(f \circ c_{M}\right)(t)=\lim _{s \rightarrow 0^{+}} f^{(n)}(s) .
$$

But $f \circ c_{M}$ is smooth, therefore $\lim _{s \rightarrow 0^{+}} f^{(n)}(s)(-M)^{n}=\lim _{s \rightarrow 0^{+}} f^{(n)}(s)$, which implies that $\lim _{s \rightarrow 0^{+}} f^{(n)}(s)=0$.

We have shown that the zero derivative property of $f$ holds for the left endpoint of the unit interval. To show that the zero derivative property of $f$ holds for the right endpoint of $f$, note that $d_{M}: \mathbb{R} \rightarrow \mathbb{R}, d_{M}(t)=1-c_{M}(t)$, is a smooth real-valued function with $d(0)=1$, and $0 \leqslant d_{M}(t) \leqslant 1$ for all $t \in \mathbb{R}$. One can follow a similar procedure to the above, using $d_{M}$ instead of $c_{M}$ to show that $\lim _{s \rightarrow 1^{-}} f^{(n)}=0$.

\subsection{Some properties of smooth functions between the flattened unit intervals}

One has to be careful when dealing with the various flattened unit intervals. A smooth function $f: I \rightarrow I$ from the $\mathbb{R}$-Frölicher subspace unit interval $I$ to itself need not define a smooth function $f: \mathbf{I} \rightarrow \mathbf{I}$, for example. Conversely, not every smooth function $f: \mathbf{I} \rightarrow \mathbf{I}$ defines a smooth function $f: I \rightarrow I$. In particular, we need to be aware of the fact that addition and multiplication of functions when defined between the various flattened unit intervals does not preserve smoothness, as is the case with the usual unit interval.

Example 2.5. The function $f: I \rightarrow I, f(t)=\frac{1}{2} t$ is clearly smooth, but the corresponding function $\mathbf{f}: \mathbf{I} \rightarrow \mathbf{I}$, given by the same formula, is not smooth. To see this, let $\alpha: \mathbb{R} \rightarrow \mathbb{R}$ be a smooth braking function with the properties that

- $\alpha(t)=-1$, for $t<-\frac{3}{4}$

- $\alpha(t)=t$, for $-\frac{1}{4}<t<\frac{1}{4}$

- $\alpha(t)=1$, for $t>\frac{3}{4}$.

Define $c: \mathbb{R} \rightarrow \mathbf{I}$ by $c(t)=1-|\alpha(t)|$. The curve $c$ is smooth everywhere except at $t=0$, where $c(0)=1$. However, every generating function $g$ on $\mathbf{I}$ is constant near 1 , and so the composite $g \circ c$ is smooth. Thus $c$ is a structure curve on $\mathbf{I}$. Now, $\mathbf{f} \circ$ $c: \mathbb{R} \rightarrow \mathbf{I}$ is given by $(\mathbf{f} \circ c)(t)=\frac{1}{2}(1-|\alpha(t)|)$. Let $h: \mathbf{I} \rightarrow \mathbb{R}$ be a structure function with the properties that

- $h(s)=0$, for $s<\frac{1}{8}$

- $h(s)=s$, for $\frac{1}{4}<s<\frac{3}{4}$

- $h(s)=1$, for $\frac{7}{8}$. 
Then $(h \circ \mathbf{f} \circ c)(t)=\frac{1}{2}(1-|\alpha(t)|)$ for $t$ near 0 , and is not smooth at $t=0$. Thus $\mathbf{f}$ does not define a smooth function from $\mathbf{I}$ to $\mathbf{I}$.

Example 2.6. The function $f: \mathbf{I} \rightarrow \mathbf{I}$, given by $f(t)=\sqrt{t}$, is smooth, but the corresponding $f: I \rightarrow I$, given by the same formula, is not smooth. This follows from the fact that $f$ is smooth on the open interval $(0,1)$, and a generating function $g$ on $\mathbf{I}$ is constant near 0 and 1 . On the side, $f: I \rightarrow I$ is not smooth, because if $c: \mathbb{R} \rightarrow I$ is a structure curve with $c(t)=t^{2}$ near $t=0$, then $(f \circ c)(t)=|t|$ near $t=0$, which is not smooth on $I$ at $t=0$.

Example 2.7. The functions $f, g: \mathbf{I}^{-} \rightarrow \mathbf{I}^{-}$, given by $f(t)=\frac{1}{2} \sqrt{t}$ and $g(t)=\frac{1}{4}$, are both smooth, but the sum $f(t)+g(t)=\frac{1}{2} \sqrt{t}+\frac{1}{4}$ is not smooth.

The following lemma follows from the definition of the Frölicher structures on the various flattened unit intervals.

Lemma 2.8. Let $f: I \rightarrow I$ be a smooth function with the properties that $f(0)=0$ and $f(1)=1$. Then the following maps are smooth:

- $f: I \rightarrow \mathbf{I}^{ \pm}$

- $f: I \rightarrow \mathbb{I}$

- $f: \mathbf{I}^{ \pm} \rightarrow \mathbf{I}$

- $f: \mathbb{I} \rightarrow \mathbf{I}$

- $f: I \rightarrow \mathbf{I}$.

The function defined in the following example is for later reference.

Example 2.9. Let $H: \mathbf{I} \times \mathbf{I}^{-} \rightarrow \mathbf{I}^{-}$be given by $H(t, s)=(1-\alpha(t)) s$, where $\alpha: \mathbb{R}$ $\rightarrow \mathbb{R}$ is a smooth braking function with the properties that

- $\alpha(t)=0$ for $t<\frac{1}{4}$

- $0 \leqslant \alpha(t) \leqslant 1$ for all $t \in \mathbb{R}$

- $\alpha(t)=1$ for $t>\frac{3}{4}$.

We show that $H$ is smooth. To see this, let $f: \mathbf{I}^{-} \rightarrow \mathbb{R}$ be a generating function on $\mathbf{I}^{-}$. So $f$ is constant near 0 . Now, let $c: \mathbb{R} \rightarrow \mathbf{I} \times \mathbf{I}^{-}$be a structure curve, given by $c(v)=(t(v), s(v))$. The curve $t$ is a structure curve on $\mathbf{I}$, and so is a smooth realvalued function for all $v \in \mathbb{R}$, except possibly when $t(v)=0$ or $t(v)=1$. Similarly, the curve $s$ is a structure curve on $\mathbf{I}^{-}$, and so is smooth for all $v \in \mathbb{R}$ except possibly when $s(v)=0$. Now consider the composite $H \circ c: \mathbb{R} \rightarrow \mathbf{I}^{-}$. Clearly, $\alpha(t(v))$ is smooth for all $v$, since the only possible points for non-smoothness occur when $t(v)=0$ or $t(v)=1$, and $\alpha(t(v))$ is locally constant near these points. Consequently, $H \circ c$ is smooth everywhere except possibly when $s(v)=0$. Now, let us consider $f \circ H \circ c: \mathbb{R} \rightarrow \mathbb{R}$; the only possible points for non-smoothness are those $v$ in which $s$ is 0 , i.e. $(H \circ c)(v)=0$. But $f$ is a structure generating function on $\mathbf{I}^{-}$, and so is locally constant near 0 . This shows that $f \circ H \circ c$ is smooth for all $v \in \mathbb{R}$, and thus $H$ is smooth. 


\subsection{Homotopy in $\mathbb{F} \mathbb{R} L$ and related objects Definition 2.10.}

1. Let $X$ be a Frölicher space, and $x_{0}, x_{1} \in X$. We say that $x_{0}$ is smoothly pathconnected to $x_{1}$ if there is a smooth path $c: I \rightarrow X$ such that $c(0)=x_{0}$ and $c(1)=x_{1}$. We write $x_{0} \simeq x_{1}$. The relation $\simeq$ is called smooth homotopy when it is applied to hom-sets.

2. Let $f: X \rightarrow Y$ be a map of Frölicher spaces. $f$ is called a smooth homotopy equivalence provided there exists a smooth map $g: Y \rightarrow X$ such that $f \circ g$ $\simeq 1_{Y}$ and $g \circ f \simeq 1_{X}$.

3. We denote the class of smooth cofibrations (defined in the following subsection) by cof and the class of smooth homotopy equivalences by we. We also call the elements of these classes the cofibrations and weak equivalences respectively, in keeping with Baues's [1] terminology.

One can show that smooth homotopy is a congruence in $\mathbb{R} F \mathbb{L}$. In practice, we say that smooth maps $f, g: X \rightarrow Y$ are smoothly homotopic if there exists a smooth map $H: I \times X \rightarrow Y$ with $H(0,-)=f$ and $H(1,-)=g$. If $A \subseteq X$ is subspace of $X$, then we say that $H$ is a smooth homotopy $(\operatorname{rel} A$ ) if the map $H$ has the additional property that $H(t, a)=a$ for each $t \in I$ and $a \in A$. See Cherenack [6] and Dugmore [7] for more detail regarding smooth homotopy.

The notion of deformation retract is fundamental to topological homotopy theory. The following definitions are adapted for smooth homotopy, and will be needed at a later stage.

Definition 2.11. Let $A \subseteq X$ be a subspace of a Frölicher space $X$, and let $i$ : $A$ $\hookrightarrow X$ denote the inclusion map. Then

1. We say that $A$ is a retract of $X$ if there exists a smooth map $r: X \rightarrow A$ such that $r i=1_{A}$. We call $r$ a retraction.

2. We call $A$ a weak deformation retract of $X$ if the inclusion $i$ is a smooth homotopy equivalence.

3. The subspace $A$ is called a deformation retract of $X$ if there exists a retraction $r: X \rightarrow A$ such that $i r \simeq 1_{X}$.

4. The subspace $A$ is called a strong deformation retract of $X$ if there exists a retraction $r: X \rightarrow A$ such that $i r \simeq 1_{X}($ rel $A)$.

Definition 2.12. The mapping cylinder $I_{f}$ of $f: X \rightarrow Y$ is defined by the following pushout

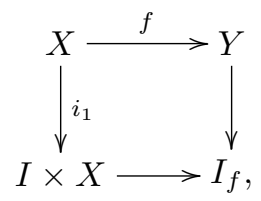

where $i_{1}: X \rightarrow I \times X$ is given by $i_{1}(x)=(1, x)$, for any $x \in X$. We denote the elements of $I_{f}$ by $[t, x]$ or $[y]$, where $(t, x) \in I \times X$ and $y \in Y$. 
Replacing $I \times X$ in the above pushout diagram by $\mathbf{I} \times X$ or $\mathbb{I} \times X$, we obtain the flattened mapping cylinder $\mathbf{I}_{f}$ and weakly flattened mapping cylinder $\mathbb{I}_{f}$ of $f$, respectively. We use the same notation for elements of these flattened mapping cylinders as described above for the mapping cylinder.

There is also a map $i_{0}: X \rightarrow I \times X$, defined by $i_{0}(x)=(0, x)$ for $x \in X$. This induces an inclusion map $i_{0}^{\prime}: X \rightarrow I_{f}$, which identifies $X$ with the Frölicher subspace $i_{0}^{\prime}(X)$ of $I_{f}$. An inclusion is induced in a similar way for the flattened mapping cylinders. If one identifies $\{0\} \times X$ to a point in the mapping cylinder $I_{f}$ of a map $f: X \rightarrow Y$, then one obtains the mapping cone $T_{f}$ of the map $f$. In a similar fashion, we define the flattened mapping cone $\mathbf{T}_{f}$ and weakly flattened mapping cone $\mathbb{T}_{f}$ of a smooth map $f: X \rightarrow Y$.

We shall need the flattened double mapping cylinder $\mathbf{I}_{i, f}$ later.

Definition 2.13. Given two smooth maps $f: X \rightarrow Y$ and $g: X \rightarrow Z$, we define the flattened double mapping cylinder $\mathbf{I}_{i, f}$ of $f$ and $g$ by the pushout

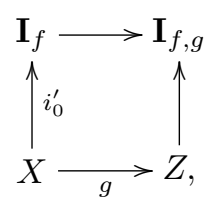

where $i$ is the inclusion into the flattened mapping cylinder.

\subsection{Cofibrations in $\mathbb{F} \mathbb{R} \mathbb{L}$}

A cofibration is a map $i: A \rightarrow X$ for which the problem of extending functions from $i(A)$ to $X$ is a homotopy problem. In other words, if a map $f: i(A) \rightarrow Z$ can be extended to a map $f^{*}: X \rightarrow Z$, then so can any map homotopic to $f$. For topological spaces, the usual definition is phrased in a slightly more restrictive way. The extension of a map $g \simeq_{H} f$, for some homotopy $H: I \times i(A) \rightarrow Z$, is required to exist at every level of the homotopy simultaneously. In other words, one requires each $H(t,-)$ to be extendable in such a way that the resulting homotopy $H^{*}: I \times X \rightarrow Z$ is continuous.

We weaken this definition somewhat, to enable smooth homotopy extensions to be more easily constructed using a flattening at the endpoints of the homotopy. This enables us to characterize smooth cofibrations in terms of a flattened unit interval, and then later to relate smooth cofibrations to smooth neighborhood deformation retracts. Our definition of smooth cofibration, though different from Cap's definition, see [2], leads to several classical results as does Cap's. As pointed out by Cap, the analogue of the classical definition of cofibration would not allow even $\{0\} \hookrightarrow I$ to be a smooth cofibration. So, we have the following 
Definition 2.14. A smooth map $i: A \rightarrow X$ is called a smooth cofibration if, corresponding to every commutative diagram of the form

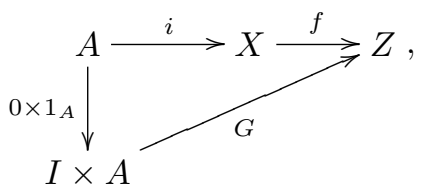

there exists a commutative diagram in $\mathbb{F R \mathbb { L }}$ of the form

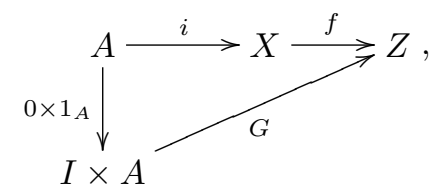

in which $G^{\prime}: I \times A \rightarrow Z$ is given by $G^{\prime}(t, a)=G\left(\alpha_{\epsilon}(t), a\right)$ for all $t \in I$ and $a \in A$. The mapping $\alpha_{\epsilon}: \mathbb{R} \rightarrow \mathbb{R}$, where $0<\epsilon<\frac{1}{2}$, is a braking function.

In the sequel, the corresponding homotopy $G^{\prime}$ of the homotopy $G: I \times A \rightarrow Z$ in a cofibration $i: A \rightarrow X$ shall mean the map given by

$$
G^{\prime}(t, a)=G\left(\alpha_{\epsilon}(t), a\right),
$$

where every $0<\epsilon<\frac{1}{2}$ and $\alpha_{\epsilon}$ is a braking function, but not necessarily a standard braking one.

Lemma 2.15. Let $i: A \rightarrow X$ be a smooth cofibration; then $i$ is an initial morphism in $\mathbb{F R L}$. In addition, if $A$ is Hausdorff, then $i$ is injective.

Proof. Let us show that every smooth map $f: A \rightarrow \mathbb{R}$ factors through $i$, that is for every $f \in \mathcal{F}_{A}$, there exists $\tilde{f} \in \mathcal{F}_{X}$ such that $f=\tilde{f} \circ i$. To this end, consider the smooth map $G: I \times A \rightarrow \mathbb{R}$, given by $G(t, a)=t f(a)$. Clearly, $\left.0\right|_{A}=G(0,-)$, where $0: X \rightarrow \mathbb{R}$ is the constant map 0 . It follows that there is map $F: I \times X \rightarrow \mathbb{R}$ such that $F \circ\left(1_{I} \times i\right)=G^{\prime}$. Then, clearly $\tilde{f}:=F(1,-)$ has the desired property.

The remaining part of the proof of Proposition 3.3 in $[\mathbf{2}]$ holds verbatim here as well.

In this paper, we are interested only in cofibrations that are injective. Henceforth, all cofibrations are assumed to be injective.

All topological cofibrations are inclusions, and this result is true for smooth cofibrations too. The proof of the following lemma is essentially the same as the proof given by James [10] for the topological result, although James's proof is in some sense dual to ours, using path-spaces in place of cartesian products and the adjoint versions of our homotopies.

Lemma 2.16. A cofibration

$$
A \stackrel{i}{\longrightarrow} X
$$

is a smooth embedding. So in this case $A$ can be identified with the subspace $i(A)$. 
Proof. Let $\mathbb{I}_{i}$ be a mapping cylinder of $i$, and let $j: X \rightarrow \mathbb{I}_{i}$ be the standard inclusion map. Consider the smooth map $\gamma: I \rightarrow I, \gamma(t)=1-t$, for all $t \in I$, and the quotient map $q:(I \times A) \sqcup X \rightarrow \mathbb{I}_{i}$. We have the following commutative diagram

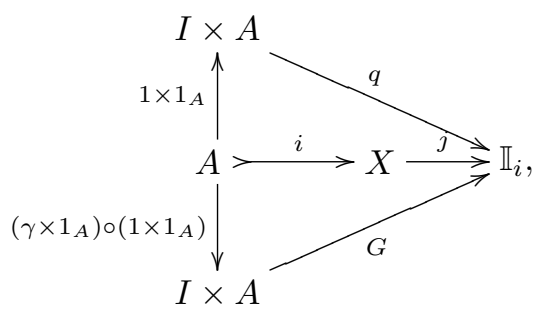

where $G(t, a)=[(1-t, a)]$. Notice that the map $G$ is smooth. Since $i$ is a cofibration, we have the commutative diagram

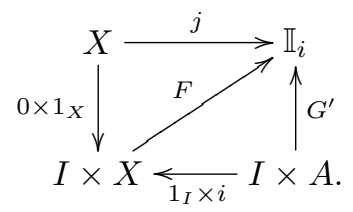

Define $U: X \rightarrow \mathbb{I}_{i}$ by $U(x)=F(1, x)$. We have $U \circ i=G^{\prime}(1,-)$, where $G^{\prime}(1, a)=$ $[(0, a)]$, for every $a \in A$. Thus, $U \circ i$ defines the usual inclusion of $A$ into the mapping cylinder $\mathbb{I}_{i}$; hence, by Lemma 1.1, $i$ is an embedding.

The result, provided in [2], that the inclusion of a unit sphere into the unit disc is a smooth cofibration also holds in our context. Cap's proof $[\mathbf{2}]$ is shortened after suitable modifications.

Example 2.17. The inclusion $i: S^{n-1} \hookrightarrow D^{n}$ is a smooth cofibration.

Proof. Consider $D^{n}$ as being embedded in the usual way in $\mathbb{R}^{n}$, and let $G: I \times$ $S^{n-1} \rightarrow Z$ and $f: D^{n} \rightarrow Z$, where $Z$ is an arbitrary Frölicher space, be smooth maps such that $G(0, x)=f(x)$, for all $x \in S^{n-1}$. Let \|\| be the usual norm on $\mathbb{R}^{n}$. Then the prescription consisting of $x \mapsto \frac{\alpha_{\epsilon}(2|| x||)}{\|x\|}$, for $x \neq 0$, and $x \mapsto 0$, for $x=0$, clearly defines a smooth real-valued function on $\mathbb{R}^{n}$. Now, define $F: I \times D^{n} \rightarrow Z$ by setting

$$
F(t, x)= \begin{cases}f\left(x \cdot\left((1-t)+t \frac{\left.\alpha_{\epsilon}(2|| x \mid)\right)}{2\|x\|}\right)\right) & \|x\| \leqslant \frac{1}{2} \\ G\left(\alpha_{\epsilon}\left(2 t-t \frac{\alpha_{\epsilon}(2|| x||)}{\|x\|}\right), x\right), & \|x\| \geqslant \frac{1}{2} .\end{cases}
$$

Since, for $\|x\|=\frac{1}{2}$ and all $t \in I$, both parts in the definition of $F$ are equal, it follows that $F$ is continuous. Moreover, since, for all $t \in I, F(t,-)$ is obviously smooth on the open disc of radius $(1+\epsilon) / 2$ and on the ring formed by all $x$ with $(1-\epsilon) / 2<$ $\|x\| \leqslant 1$, it follows that $F(t,-)$ is smooth all over $D^{n}$, and hence $F: I \times D^{n} \rightarrow Z$ is smooth.

It remains only to verify that $F(0, x)=f(x)$, for all $x \in D^{n}$, and $F(t, x)=$ $G\left(\alpha_{\epsilon}(t), x\right)$, for all $t \in I$ and $x \in S^{n-1}$, which is obviously easy to show. 
There is an equivalent formulation of Definition 2.14, given in the following lemma.

Lemma 2.18. A smooth map

$$
A>\stackrel{i}{\longrightarrow} X
$$

is a cofibration if and only if, for every smooth map $h:(0 \times X) \cup\left(\mathbf{I}^{-} \times i(A)\right) \rightarrow Z$, the following diagram

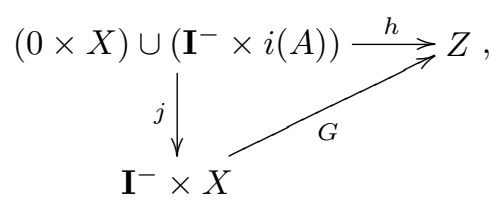

where $j$ is the evident inclusion, exists in $\mathbb{F} \mathbb{R L}$.

Proof. Suppose that the inclusion $A>\stackrel{i}{\longrightarrow} X$ is a smooth cofibration, and suppose that $h:(0 \times X) \cup\left(\mathbf{I}^{-} \times i(A)\right) \rightarrow Z$ is a smooth map. We have the diagram

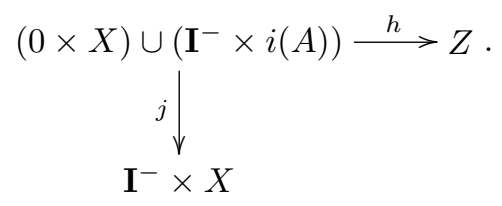

We need to fill in a smooth map $G: \mathbf{I}^{-} \times X \rightarrow Z$ which makes the resulting diagram commute. To do this, notice that $\left.h\right|_{\mathbf{I}^{-} \times i(A)}$ is smooth, and thus the corresponding map $\left.h\right|_{I \times i(A)}$, using the usual unit interval, is also smooth. We have the following diagram

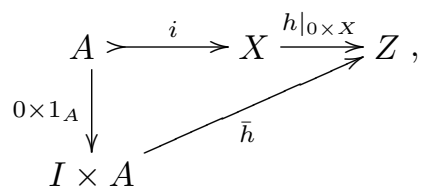

where $\bar{h}=h \circ\left(1_{I} \times i\right)$, and $h(0,-): X \rightarrow Z$ is denoted as $\left.h\right|_{0 \times X}$. The fact that $i$ is a smooth cofibration yields the following $\mathbb{F} \mathbb{R} L$-commutative diagram:

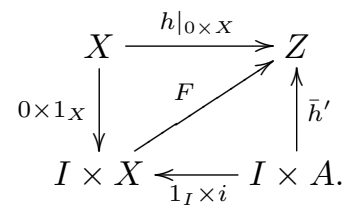

Now, choose a smooth braking function $\beta: \mathbb{R} \rightarrow \mathbb{R}$ with the following properties:

- $\beta(t)=0$ for $t \leqslant \frac{\epsilon}{2}$

- $0<\beta(t)<\epsilon$ for $\frac{\epsilon}{2}<t<\epsilon$

- $\beta(t)=t$ for $\epsilon \leqslant t$. 
$F$ may not be smooth on $\mathbf{I}^{-} \times X$ due to the flattening requirements of the left flattened unit interval. To correct this, set $G(t, x)=F(\beta(t), x)$. Notice that the insertion of this braking function does not affect the commutativity conditions of $G$, since the only adjustments to $F$ occur in the first coordinate at points where the map $\bar{h}^{\prime}$ is constant.

Now, assume the converse; i.e., to every smooth $h:(0 \times X) \cup\left(\mathbf{I}^{-} \times i(A)\right) \rightarrow Z$, there corresponds a commutative diagram

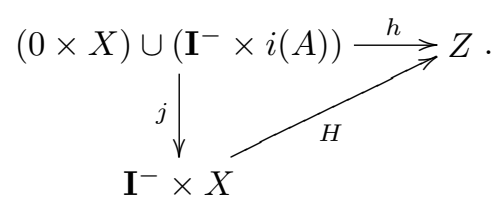

We wish to show that the inclusion $i: A \rightarrow X$ is a cofibration; so assume we have the following diagram

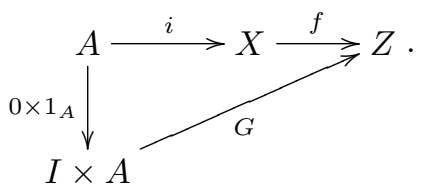

Since $i$ is injective, there exists the diagram

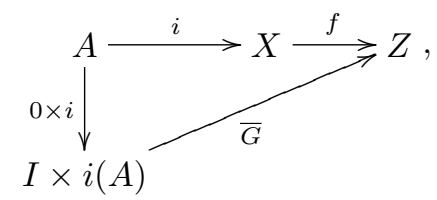

where $\bar{G}(t, i(a))=G\left(\alpha_{\epsilon}(t), a\right)$. We denote by $G^{*}$ the mapping $\mathbf{I}^{-} \times i(A) \rightarrow Z$ that corresponds to $\bar{G}: I \times i(A) \rightarrow Z$; it is clear that $G^{*}: \mathbf{I}^{-} \times i(A) \rightarrow Z$ is smooth. Putting $f=f \circ\left(0 \times 1_{X}\right)$, and since $f \circ G^{*}:(0 \times X) \cup\left(\mathbf{I}^{-} \times i(A)\right) \rightarrow Z$ is smooth, our hypothesis allows us to construct the diagram

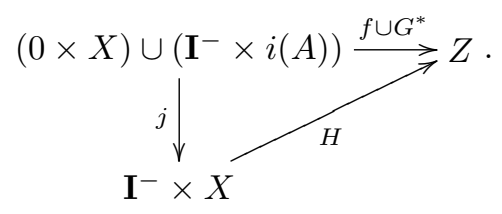

Since $H$ is smooth on $\mathbf{I}^{-} \times X$, it defines a smooth map on $I \times X$. One can verify that the diagram

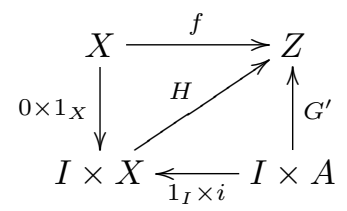

commutes as required. 


\section{Smooth neighborhood deformation retracts}

This section is concerned with the formulation of a suitable notion of smooth neighborhood deformation retract. For topological spaces, the statement that a closed subspace $A$ of $X$ is a neighborhood deformation retract of $X$ is equivalent to the statement that the inclusion $i: A \hookrightarrow X$ is a closed cofibration. We show that in the category of Frölicher spaces there is a notion of smooth neighborhood deformation retract that gives rise to an analogous result that a closed Frölicher subspace $A$ of the Frölicher space $X$ is a smooth neighborhood deformation retract of $X$ if and only if the inclusion $i: A \hookrightarrow X$ comes from a certain subclass of cofibrations. As an application, we construct the right Puppe sequence.

\subsection{SNDR pairs and SDR pairs}

The definition of 'smooth neighborhood deformation retract' that we adopt in this paper is similar to the definition of 'R-SNDR pair' suggested in [7], but we have modified the definition in order to retain only the essential aspects of 'first coordinate independence' defined in [7].

We begin by defining the 'first coordinate independence property' of a function on a product of a Frölicher space with $\mathbf{I}\left(\right.$ or $\left.\mathbf{I}^{-}, \mathbf{I}^{+}\right)$.

Definition 3.1. Let $i: A \rightarrow X$ be a smooth map, and $c: \mathbb{R} \rightarrow X$ a structure curve on $X$. Define

$$
\begin{aligned}
\Lambda(c, i)= & \left\{t_{*} \in c^{-1}(i(A)) \mid \text { there exists a sequence }\left\{t_{n}\right\}\right. \text { of real numbers } \\
& \text { with } \left.\lim _{n \rightarrow \infty} t_{n}=t_{*} \text { and each } t_{n} \in c^{-1}(X-i(A))\right\} .
\end{aligned}
$$

The points in $\Lambda(c, i)$ are those values in $\mathbb{R}$ where the curve 'enters' $i(A)$ from $X-i(A)$, or 'touches' a point in $i(A)$ whilst remaining in $X-i(A)$ nearby. Now, we are ready to define the 'first coordinate independence property' for a structure function on a product.

Definition 3.2. Let $i: A \rightarrow X$ be a smooth map and suppose $f: \mathbf{I} \times X \rightarrow \mathbb{R}$ is a structure function on $\mathbf{I} \times X$. Let $c: \mathbb{R} \rightarrow \mathbf{I} \times X$, given by $c(s)=(t(s), x(s))$ have the following properties:

1. The map $x(s)$ is a structure curve on $X$.

2. For all $\epsilon>0, t(s)$ is a smooth real function on $\mathbb{R}-\cup_{s_{*} \in \Lambda(x, i)}\left[s_{*}-\epsilon, s_{*}+\epsilon\right]$.

If, for every such map $c$, the composite $f \circ c$ is a smooth real-valued function, then we say that $f: \mathbf{I} \times X \rightarrow \mathbb{R}$ has the first independence property (FCIP) with respect

to $i$.

Extending the definition, we say that a map $g: \mathbf{I} \times X \rightarrow Y$ has the FCIP with respect to $i$ if the composite $h \circ g: \mathbf{I} \times X \rightarrow \mathbb{R}$ has the FCIP with respect to $i$ for every $h \in \mathcal{F}_{Y}$.

Notice that we can formulate a similar definition of the FCIP if we replace $\mathbf{I}$ throughout by $\mathbf{I}^{-}$or $\mathbf{I}^{+}$, leaving the rest of the definition unchanged. We will have occasion to use this type of first coordinate independence property in the later part of this work. 
Remark 3.3. Let $i: A \rightarrow X$, and suppose that we are given a map $g: \mathbf{I} \times X \rightarrow Y$. Let $f: Y \rightarrow \mathbb{R}$ be a structure function on $Y$, and suppose that $f \circ g: \mathbf{I} \times X \rightarrow \mathbb{R}$ has the FCIP with respect to $i$ for any such $f$. Then, given a smooth map $h: Y \rightarrow Z$, the composite $f^{\prime} \circ h \circ g: \mathbf{I} \times X \rightarrow \mathbb{R}$ has the FCIP with respect to $i$ for any structure function $f^{\prime}$ on $Z$.

The above note applies equally well if $g: \mathbf{I}^{-} \times X \rightarrow Y$ or $g: \mathbf{I}^{+} \times X \rightarrow Y$ has the FCIP with respect to $i$ when composed with a smooth function $h$ on $Y$.

Example 3.4.

1. For any $i: A \rightarrow X$, the projection onto the second coordinate $\pi_{X}: \mathbf{I} \times X \rightarrow X$ has the FCIP.

2. Let $\alpha: \mathbb{R} \rightarrow \mathbb{R}$ be a smooth braking function with the properties that

- $\alpha(t)=0$ if $t<\frac{1}{4}$

- $0<\alpha(t)<1$ if $\frac{1}{4} \leqslant t \leqslant \frac{3}{4}$

- $\alpha(t)=1$ if $\frac{3}{4}<t$.

Consider $0 \hookrightarrow \mathbf{I}^{-}$. Let $H: \mathbf{I} \times \mathbf{I}^{-} \rightarrow \mathbf{I}^{-}$be given by $H(t, s)=(1-\alpha(t)) s$. Then, $f \circ H: \mathbf{I} \times \mathbf{I}^{-} \rightarrow \mathbb{R}$ has the FCIP with respect to the inclusion $0 \hookrightarrow \mathbf{I}^{-}$, for any $f \in \mathcal{F}_{\mathbf{I}^{-}}$.

Definition 3.5. Consider a smooth inclusion $i: A \hookrightarrow X$. Suppose that there exists a smooth map $u: X \rightarrow \mathbf{I}$, with $u^{-1}(0)=i(A)$. If there exists a smooth map $H: \mathbf{I} \times$ $X \rightarrow X$ that satisfies the following properties:

1. $H$ has the FCIP with respect to $i$.

2. $H(0, x)=x$ for all $x \in X$

3. $H(t, x)=x$ for all $(t, x) \in I \times i(A)$

4. $H(1, x) \in i(A)$ for all $x \in X$ with $u(x)<1$.

Then the pair $(X, A)$ is called a smooth neighborhood deformation retract pair, or SNDR pair for short.

If, in addition, $H$ is such that $H(1 \times X) \subset i(A)$, then the pair $(X, A)$ is called a smooth deformation retract pair, or an SDR pair for short.

The subspace $A$ is called a smooth neighborhood deformation retract or smooth deformation retract of $X$ if $(X, A)$ is an SNDR pair or SDR pair, respectively.

The pair $(u, H)$ is called a representation for the SNDR (or SDR) pair.

Example 3.6.

1. The pair $(X, \emptyset)$ is an SNDR pair. A representation is $u(x)=1, H(t, x)=x$, for each $t \in \mathbf{I}$ and $x \in X$.

2. The pair $(X, X)$ is an SNDR pair. A representation is $u(X)=0, H(t, x)=x$, for each $t \in \mathbf{I}$ and $x \in X$.

Lemma 3.7. The pair $\left(\mathbf{I}^{-}, 0\right)$ is an SDR pair.

Proof. Let $\alpha: \mathbb{R} \rightarrow \mathbb{R}$ be the smooth braking function of Examples 3.4. A representation for $\left(\mathbf{I}^{-}, 0\right)$ as an SDR pair is $(u, H)$, where $u: \mathbf{I}^{-} \rightarrow \mathbf{I}$ and $H: \mathbf{I} \times \mathbf{I}^{-} \rightarrow \mathbf{I}^{-}$ 
are given by $u(s)=s$, and $H(t, s)=(1-\alpha(t)) s$. Clearly, the identity $u: \mathbf{I}^{-} \rightarrow \mathbf{I}$ is smooth. And the map $H$, as shown in Example 2.9, is smooth and clearly has the FCIP with respect to the inclusion, since whenever $v$ approaches a value for which $s(v)=0$, one has

$$
g((1-\alpha(t(v))) s(v))=g(0)
$$

for $v$ in a neighborhood of this value and $g \in \mathcal{F}_{\mathbf{I}^{-}}$.

Lemma 3.8. The pair $(\mathbf{I},\{0,1\})$ is an SNDR pair.

Proof. A representation $(u, H)$ for the SNDR pair can be given as follows. Define $u: \mathbf{I} \rightarrow \mathbf{I}$ to be a bump function such that

- $u(t)=0$ for $t=0$ or $t=1$

- $u(t)=1$ for $t \in\left[\frac{1}{4}, \frac{3}{4}\right]$

- $0<u(t)<1$ otherwise,

and let $\beta: \mathbf{I} \rightarrow \mathbf{I}$ be a braking function with the properties that $\beta(s)=0$ for $0 \leqslant s$ $\leqslant \frac{1}{4}$, and $\beta(s)=1$ for $\frac{3}{4} \leqslant s \leqslant 1$. Let $0<\epsilon<\frac{1}{4}$, and define $H: \mathbf{I} \times \mathbf{I} \rightarrow \mathbf{I}$ by $H(t, s)$ $=\left(1-\alpha_{\epsilon}(t)\right) s+\alpha_{\epsilon}(t) \beta(s)$. It is clear that $H(0, s)=s, H(t, 0)=0$, and $H(t, 1)=1$. Suppose that $u(s)<1$. Then, $s \in\left[0, \frac{1}{4}\right) \cup\left(\frac{3}{4}, 1\right]$. This implies that $\beta(s)=0$ or $\beta(s)$ $=1$. We then have $H(1, s)=0$ or $H(1, s)=1$, which means that $H(1, s) \in\{0,1\}$ if $u(s)<1$.

To see that $H$ is smooth, let $f: \mathbf{I} \rightarrow \mathbb{R}$ be a generating function for the flattened unit interval. The only possible points of non-smoothness are points where $t=0,1$ and $s=0,1$. The braking function $\alpha_{\epsilon}$ ensures that $H$ is locally constant in the $t$ variable whenever $t$ is near 0 or 1 , so no problem arises from the $t$ component. When $s$ is near $s=0$, we have $H(t, s)$ near 0 , and so $f \circ H$ is locally constant near any $(t, 0)$. Similarly, when $s$ is near $s=1$, we have $H(t, s)$ near 1 , and $f \circ H$ is again locally constant near any $(t, 1)$.

We now show that the product of SNDR pairs is again an SNDR pair.

Theorem 3.9. Let $i: A \hookrightarrow X$ and $j: B \hookrightarrow Y$ be inclusion mappings. If $(X, A)$ and $(Y, B)$ are SNDR pairs, then so is

$$
(X \times Y,(X \times B) \cup(A \times Y)) .
$$

If one of $(X, A)$ or $(Y, B)$ is an SDR pair, then so is the pair

$$
(X \times Y,(X \times B) \cup(A \times Y)) .
$$

Proof. Let $\alpha: \mathbb{R} \rightarrow I$ be a smooth braking function with the properties that $\alpha(t)=0$ for $t \leqslant \frac{1}{4}$, and $\alpha(t)=1$ for $t \geqslant \frac{3}{4}$, and let $\beta: \mathbb{R} \rightarrow \mathbb{R}$ be a smooth increasing braking function with the properties that $\beta(t)=t$ for $t \leqslant \frac{1}{4}$, and $\beta(t)=1$ for $t \geqslant \frac{3}{4}$. Suppose that $(u, H)$ and $(v, J)$ are representations for the SNDR pairs $(X, A)$ and $(Y, B)$, respectively. Let $\bar{u}: X \rightarrow \mathbf{I}$, and $\bar{v}: Y \rightarrow \mathbf{I}$ be given by $\bar{u}(x)=\beta(u(x))$ and $\bar{v}(y)=\beta(v(y))$ respectively. Define $w: X \times Y \rightarrow \mathbf{I}$ by $w(x, y)=\bar{u}(x) \bar{v}(y) ; w$ is 
clearly smooth. We have $w^{-1}(0)=(X \times B) \cup(A \times Y)$, as required. Define $Q: \mathbf{I} \times$ $X \times Y \rightarrow X \times Y$ as follows:

$$
Q(t, x, y)= \begin{cases}(H(\alpha(t), x), J(\alpha(t), y)) & \text { if } \bar{u}(x)=\bar{v}(y)=0 \\ \left(H(\alpha(t), x), J\left(\alpha\left(\frac{\bar{u}(x)}{\bar{v}(y)}\right) \alpha(t), y\right)\right) & \text { if } \bar{v}(y) \geqslant \bar{u}(x), \bar{v}(y)>0 \\ \left(H\left(\alpha\left(\frac{\bar{v}(y)}{\bar{u}(x)}\right) \alpha(t), x\right), J(\alpha(t), y)\right) & \text { if } \bar{u}(x) \geqslant \bar{v}(y), \bar{u}(x)>0\end{cases}
$$

We must show that $Q$ is a smooth map, with the first coordinate independence property with respect to the inclusion $(X \times B) \cup(A \times Y) \hookrightarrow X \times Y$. We first consider each part of the definition of $Q$ separately. The first part is clearly smooth. Let us verify that $Q$ is smooth on the second part of its definition; the third part is similar.

We need only focus on the component $J\left(\alpha\left(\frac{\bar{u}(x)}{\bar{v}(y)}\right) \alpha(t), y\right)$. Each function making up $J\left(\alpha\left(\frac{\bar{u}(x)}{\bar{v}(y)}\right) \alpha(t), y\right)$ is smooth individually, so we need only pay extra attention to those parts that involve flattened unit intervals, remembering that addition and multiplication on the flattened unit interval need not preserve smoothness, as is the case for the usual unit interval.

So let us consider $\alpha\left(\frac{\bar{u}(x)}{\bar{v}(y)}\right)$; it is smooth except possibly when $\frac{\bar{u}(x)}{\bar{v}(y)}$ approaches 0 or 1 , since it is here that structure curves on the flattened unit interval need not be smooth in the usual sense. Clearly, if $u(x)$ approaches 0 and $v(y)$ does not approach 0 , then the braking function $\alpha$ ensures that $\frac{\bar{u}(x)}{\bar{v}(y)}=0$ near such points. If $\bar{v}(y)$ approaches 0 , then $\bar{u}(x)$ must approach 0 too. This situation is dealt with later.

Thus, $Q$, in part two of the definition, is smooth, and one can show similarly that $Q$ in the third part of the definition is smooth as well.

Let us now consider the overlaps of the three parts of the definition of $Q$. Observe that if $\bar{u}(x)$ is in a sufficiently small neighborhood of $\bar{v}(y)$, with $\bar{u}(x) \neq 0$ and $\bar{v}(y)$ $\neq 0$, then we have $\alpha\left(\frac{\bar{u}(x)}{\bar{v}(y)}\right)=\alpha\left(\frac{\bar{v}(y)}{\bar{u}(x)}\right)=1$, and so the second and third parts of the definition of $Q$ coincide here. Thus, it remains only to show that $Q$ is smooth as $\bar{u}(x)$ and $\bar{v}(y)$ both approach 0 .

If $Q$ is smooth in each of its coordinates then it is smooth, so consider the coordinate involving the map $J$. Let $c: \mathbb{R} \rightarrow \mathbf{I} \times X \times Y$ be a structure that is given by $c(s)=(t(s), x(s), y(s))$. Then, the map $c_{1}: \mathbb{R} \rightarrow \mathbf{I} \times Y$, given by

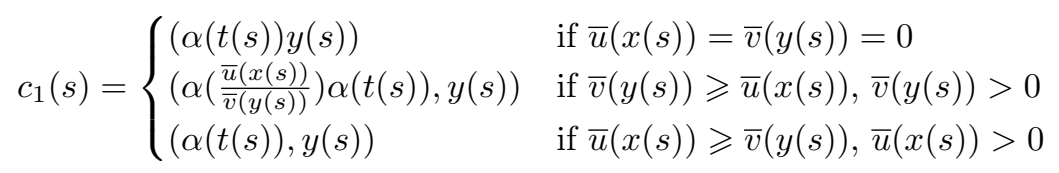

is a map satisfying the conditions of Definition 3.2, since its second coordinate is smooth, but its first coordinate may be singular as $\bar{v}(y(s)$ ) (and hence $\bar{u}(x(s))$ ) approaches 0 . Since $J$ has the first coordinate independence property, the map

$$
\left(J_{o c_{1}}\right)(s)= \begin{cases}J(\alpha(t(s)), y(s)) & \text { if } \bar{u}(x(s))=\bar{v}(y(s))=0 \\ J\left(\alpha\left(\frac{\bar{u}(x(s))}{\bar{v}(y(s))}\right) \alpha(t(s)), y(s)\right) & \text { if } \bar{v}(y(s)) \geqslant \bar{u}(x(s)), \bar{v}(y(s))>0 \\ J(\alpha(t(s)), y(s)) & \text { if } \bar{u}(x(s)) \geqslant \bar{v}(y(s)), \bar{u}(x(s))>0\end{cases}
$$


is smooth. In a similar way, the coordinate of $Q$ involving $H$ can be shown to be smooth as $\bar{u}(x)$, and $\bar{v}(y)$ approach 0 . Thus, $Q \circ c$ is smooth, and since $c$ is arbitrary, $Q$ is smooth.

We now verify that $Q$ satisfies the required boundary conditions. When $t=0$, all three lines defining $Q$ reduce to $(H(0, x), J(0, y))=(x, y)$. Let $x \in A$ and $y \in B$; then $\bar{u}(x)=\bar{v}(y)=0$. Therefore, $Q$ reduces to $(H(\alpha(t), x), J(\alpha(t), y))=(x, y)$. If $x \in A$ and $y \notin B$, then $Q$ is given by the second part of its definition, which reduces to $(H(\alpha(t), x), J(0, y))$. The case when $x \notin A$ and $y \in B$ is similar. If $t=1$ and $0<w(x, y)<1$, then $0<\bar{u}(x)<1$ and $0<\bar{v}(y)<1$. Suppose that $0<\bar{u}(x)<1$. Then either $\bar{u}(x) \leqslant \bar{v}(y)$ or $\bar{v}(y)<\bar{u}(x)$. If $\bar{u}(x) \leqslant \bar{v}(y)$, then $Q$ is given by the second part of its definition, which reduces to $\left(H(1, x), J\left(\alpha\left(\frac{\bar{u}(x)}{\bar{v}(y)}\right), y\right)\right) \in i(A) \times Y$. If $\bar{v}(y)<\bar{u}(x)$, then the third part of the definition of $Q$ applies and $Q$ reduces to $\left(H\left(\alpha\left(\frac{\bar{v}(y)}{\bar{u}(x)}\right), x\right), J(1, y)\right) \in X \times j(B)$.

Finally, we must show that for any $f \in \mathcal{F}_{X \times Y}, f \circ Q$ has the first coordinate independence property with respect to the inclusion $(X \times B) \cup(A \times Y) \hookrightarrow X \times Y$. To this end, consider a map $c: \mathbb{R} \rightarrow \mathbf{I} \times X \times Y$, given by $c(s)=(t(s), x(s), y(s))$. Let $\left\{s_{n}\right\}$ be a sequence of real numbers converging to $s_{*}$ with $c\left(s_{n}\right) \in(X \times Y)$ $((A \times Y) \cup(X \times B))$, and $c\left(s_{*}\right) \in(A \times Y) \cup(X \times B)$. There are three cases to consider.

1. Suppose that $c\left(s_{*}\right) \in A \times B$. Then $x\left(s_{*}\right) \in A$ and $y\left(s_{*}\right) \in B$. The fact that $H$ and $J$ have the first coordinate independence property with respect to $i$ and $j$ respectively means that each coordinate of $Q$ is smooth, and so $Q$ is smooth at $c\left(s_{*}\right)$.

2. Suppose that $c\left(s_{*}\right) \in A \times Y$, and that $y\left(s_{*}\right) \notin B$. Then at each of the points $c\left(s_{n}\right),(Q \circ c)\left(s_{n}\right)$ is given by the second part of the definition of $Q$, for $n$ large enough. Since $x\left(s_{*}\right) \in A$, the component of $Q$ involving $H$ is smooth, since $H$ has the first coordinate independence property. For any $s$ in a neighborhood of $s_{*}, \alpha\left(\frac{\bar{u}(x(s))}{\bar{v}(y(s))}\right)=0$. Thus, the component of $Q$ involving $J$ is constant for $s$ in a neighborhood of $s_{*}$, and so is smooth there.

3. The case with $c\left(s_{*}\right) \in X \times B$, and $x\left(s_{*}\right) \notin A$ is similar to the second case above.

For the last part of the theorem, suppose that $(u, H)$ represent $(X, A)$ as an SDR pair. If we replace $u$ by $u^{\prime}=\frac{1}{2} u$, then $\left(u^{\prime}, H\right)$ also represent $(X, A)$ as an SDR pair. Making the above constructions now with $u^{\prime}$ in place of $u$, it follows that $w(x, y)<1$ for all $(x, y)$ and so $Q(1, x, y) \in(X \times B) \cup(A \times Y)$. This completes the proof.

\section{Cofibrations with FCIP}

In this section, we show that for a subspace $A \subseteq X$ that is closed in the underlying topology, the inclusion $i: A \rightarrow X$ is a cofibration if and only if $(X, A)$ is an SNDR pair.

Definition 4.1. Let $i: A \rightarrow X$ be a cofibration. We call $i$ a cofibration with FCIP if any homotopy extension can be chosen to have the FCIP with respect to $i$. 
Using the equivalent formulation of the notion of cofibration, which is given by Lemma 2.18, we may restate Definition 4.1 as follows: A cofibration $i: A \rightarrow X$ is a cofibration with the FCIP if and only if the map $G$ that we may fill in to complete the commutative diagram,

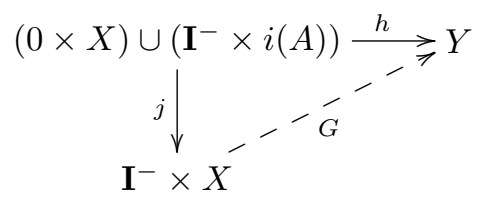

may be chosen to have the FCIP with respect to the inclusion $i$.

We have the following result, which corresponds to a similar topological result.

Lemma 4.2. A smooth map $i: A \rightarrow X$ is a cofibration (with the FCIP) if and only if $(0 \times X) \cup\left(\mathbf{I}^{-} \times i(A)\right)$ is a retract of $\mathbf{I}^{-} \times X$ (where the retraction $r: \mathbf{I}^{-} \times X \rightarrow$ $(0 \times X) \cup\left(\mathbf{I}^{-} \times i(A)\right)$ has the FCIP $)$.

Proof. In the one direction, suppose that $(0 \times X) \cup\left(\mathbf{I}^{-} \times i(A)\right)$ is a retract of $\mathbf{I}^{-}$ $\times X$. For every map $h:(0 \times X) \cup\left(\mathbf{I}^{-} \times i(A)\right) \rightarrow Y$, we wish to complete the following diagram:

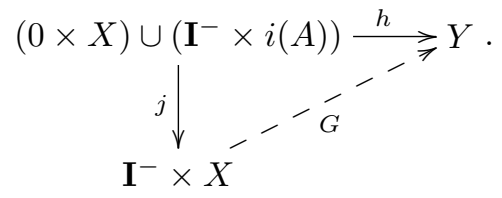

By hypothesis, there exists $r: \mathbf{I}^{-} \times X \rightarrow(0 \times X) \cup\left(\mathbf{I}^{-} \times i(A)\right)$ such that $r \circ j=1$. Define $G=h \circ r$. If $r$ has the FCIP, then so does $h \circ r$.

Conversely, suppose that $i: A \rightarrow X$ is a cofibration (with the FCIP). Define $r: \mathbf{I}^{-} \times X \rightarrow(0 \times X) \cup\left(\mathbf{I}^{-} \times i(A)\right)$ by the prescription: $r(t, x)=(t, i(a))$ if $x=$ $i(a)$, and $r(t, x)=(0, x)$ for all $x \notin i(A)$. Thus, $r \circ j=1$. If $i$ is a cofibration with the FCIP, then $r$ has also the FCIP property.

The next theorem shows the relationship between cofibrations, retracts and SNDR pairs.

Theorem 4.3. Let $i: A \rightarrow X$ be an inclusion, with $A$ closed in the underlying topology of $X$. Then the following are equivalent.

1. The pair $(X, A)$ is an SNDR pair.

2. There is a smooth retraction $r: \mathbf{I}^{-} \times X \rightarrow(0 \times X) \cup\left(\mathbf{I}^{-} \times i(A)\right)$ with the FCIP.

3. The map $i: A \rightarrow X$ is a cofibration with the FCIP.

Proof. To show that (1) and (2) are equivalent, note that the pair

$$
\left(\mathbf{I}^{-} \times X,(0 \times X) \cup\left(\mathbf{I}^{-} \times i(A)\right)\right)
$$

is an SDR pair, as a consequence of Lemma 3.7 and Theorem 3.9. Let $(w, Q)$ be a representation for the pair $\left(\mathbf{I}^{-} \times X,(0 \times X) \cup\left(\mathbf{I}^{-} \times i(A)\right)\right)$ as an SDR pair, and let 
$Q$ be constructed as in Theorem 3.9. Define

$$
r: \mathbf{I}^{-} \times X \rightarrow(0 \times X) \cup\left(\mathbf{I}^{-} \times i(A)\right)
$$

by $r(t, x)=Q(1, t, x)$, where $(t, x) \in \mathbf{I}^{-} \times X$. We observe that $r$ has the FCIP, since $Q$ has this property, and $Q$ has this property since each of its components has this property.

The equivalence of (2) and (3) is Lemma 4.2.

We need only show that (2) implies (1). Let $r: \mathbf{I}^{-} \times X \rightarrow(0 \times X) \cup\left(\mathbf{I}^{-} \times i(A)\right)$ be a retraction with the FCIP with respect to $i$. Define $H: \mathbf{I} \times X \rightarrow X$ by $H(t, x)=$ $\left(\pi_{X} \circ r\right)(\alpha(t), x)$, where $\pi_{X}$ is the projection onto the second coordinate, and $\alpha: \mathbb{R}$ $\rightarrow \mathbb{R}$ is a braking function with the following properties: $\alpha(t)=0$ for $t \leqslant 0, \alpha(t)=1$ for $t \geqslant \frac{3}{4}$, and $0<\alpha(t)<1$ for $0<t<\frac{3}{4}$. This braking function is necessary to ensure smoothness at the right endpoint of the flattened unit interval I. Smoothness at the left endpoint is already taken care of by the fact that $r$ is defined in terms of the left flattened unit interval. The map $H$ satisfies the following properties:

- $H$ has the FCIP since $r$ has this property.

- $H(0, x)=\left(\pi_{X} \circ r\right)(0, x)=x$, for $x \in X$.

- $H(t, x)=\left(\pi_{X} \circ r\right)(\alpha(t), x)=x$, for $x \in A$.

We now construct $u: X \rightarrow \mathbf{I}$. Let $\pi_{\mathbf{I}}: \mathbf{I} \times X \rightarrow \mathbf{I}$ denote the projection onto $\mathbf{I}$. Define a smooth function $\beta: \mathbb{R} \rightarrow \mathbb{R}$ by

$$
i \beta(t)= \begin{cases}0 & \text { if } t \leqslant 0 \\ e^{-\frac{1}{t^{2}}} & \text { if } t>0\end{cases}
$$

Now, define $u: X \rightarrow \mathbf{I}$ by

$$
u(x)=\frac{\int_{0}^{1} \beta\left(\alpha(t)-\left(\pi_{\mathbf{I}} \circ r\right)(1, x)\left(\pi_{\mathbf{I}} \circ r\right)(\alpha(t), x)\right) d t}{\int_{0}^{1} \beta(\alpha(t)) d t} .
$$

It is clear that $u$ is a smooth mapping.

We now verify that $(u, H)$ represents $(X, A)$ as an SNDR pair.

(A) Let $x \in A$. Clearly, $\left(\pi_{\mathbf{I}} \circ r\right)(1, x)=1$ and $\left(\pi_{\mathbf{I}} \circ r\right)(\alpha(t), x)=\alpha(t)$, and so $\int_{0}^{1} \beta\left(\alpha(t)-\left(\pi_{\mathbf{I}} \circ r\right)(1, x)\left(\pi_{\mathbf{I}} \circ r\right)(\alpha(t), x)\right) d t=0$. Thus, $u(x)=0$, for all $x \in A$.

(B) Suppose that $x \in X-A$. Since $0 \times(X-A)$ is open in the underlying topology on $(0 \times X) \cup\left(\mathbf{I}^{-} \times A\right)$, we may choose an open neighborhood $W \subseteq 0 \times(X-A)$ of $(0, x)$. Since $r$ is continuous, there is a neighborhood $V \subseteq \mathbf{I}^{-} \times X$ such that $r(V) \subseteq W \subseteq 0 \times(X-A)$. Now, consider the mapping $q_{x}: \mathbf{I} \rightarrow \mathbf{I} \times X$, given by $q_{x}(t)=(\alpha(t), x)$, for each $x \in X$. This is clearly smooth. Thus, there exists a neighborhood $U \subseteq \mathbf{I}^{-}$such that $q_{x}(U) \subseteq V$. In other words, $U \times\{x\} \subseteq V$. So, we have $\left(\pi_{\mathbf{I}} \circ r\right)(\alpha(t), x)=0$, for all $t \in U$. Thus, we have

$$
u(x)=\frac{\int_{I-U} \beta\left(\alpha(t)-\left(\pi_{\mathbf{I}} \circ r\right)(1, x)\left(\pi_{\mathbf{I}} \circ r\right)(\alpha(t), x)\right) d t+\int_{U} \beta(\alpha(t)) d t}{\int_{0}^{1} \beta(\alpha(t)) d t}>0 .
$$

Combining this with part (1), we deduce that $u^{-1}(0)=A$. 
(C) Suppose that $x$ is such that $u(x)<1$. There must be a neighborhood $U$ of $\mathbf{I}$ such that $\left(\pi_{\mathbf{I}} \circ r\right)(1, x)\left(\pi_{\mathbf{I}} \circ r\right)(\alpha(t), x)>0$, for $t \in U$. Thus $\left(\pi_{\mathbf{I}} \circ r\right)(1, x)>0$, but this implies that $r(1, x) \in \mathbf{I} \times A$, and hence $H(1, x) \in A$. The proof is complete.

\section{The mapping cylinder}

In this section we show that the inclusion of $X$ into the flattened mapping cylinder $\mathbf{I}_{f}$ of a map $f: X \rightarrow Y$ is a cofibration with the FCIP.

Theorem 5.1. Let $f: X \rightarrow Y$ be a smooth map. Then, the pair $\left(\mathbf{I}_{f}, X\right)$ is an SNDR pair.

Proof. Let $\alpha: \mathbf{I} \rightarrow \mathbb{R}$ be a smooth braking function with the following properties: $\alpha(t)=0$ if $0 \leqslant t \leqslant \frac{1}{4}, \alpha(t)=1$ if $\frac{3}{4} \leqslant t \leqslant 1,0<\alpha(t)<1$, otherwise. Define two more braking functions $\alpha_{1}, \alpha_{2}: \mathbf{I} \rightarrow \mathbb{R}$ as follows: $\alpha_{1}(0)=0,0<\alpha_{1}(t)<1$ if $0<t<\frac{3}{4}$, $\alpha_{1}(t)=1$ if $\frac{3}{4} \leqslant t \leqslant 1$, and $\alpha_{2}(t)=0$ if $0 \leqslant t \leqslant \frac{3}{4}, \alpha_{2}(t)=1$ if $\frac{7}{8} \leqslant t \leqslant 1$. Now, define $u: \mathbf{I}_{f} \rightarrow \mathbf{I}$ by $u([t, x])=\alpha_{1}(t)$ and $u([y])=1$, for $(t, x) \in \mathbf{I} \times X$ and $y \in Y$. Define $H: \mathbf{I} \times \mathbf{I}_{f} \rightarrow \mathbf{I}_{f}$ by

$$
\begin{cases}H(s,[t, x])=\left[(1-\alpha(s)) t+\alpha(s) \alpha_{2}(t), x\right] & \text { if }(t, x) \in \mathbf{I} \times X \\ H(s,[y])=[y] & \text { if } y \in Y .\end{cases}
$$

That $u$ is smooth comes from the fact that $u \circ q$, where $q$ is the quotient map $q:(\mathbf{I} \times X) \sqcup Y \rightarrow \mathbf{I}_{f}$, is smooth when restricted to each component of the coproduct $(\mathbf{I} \times X) \sqcup Y$.

To see that the map $H: \mathbf{I} \times \mathbf{I}_{f} \rightarrow \mathbf{I}_{f}$ is smooth, note that since we are working in a cartesian closed category, products commute with quotients, i.e. if $q$ is a quotient, then so is $1 \times q$, where 1 is an identity map. Thus, we may think of $H$ as being defined on the space

$$
\frac{(\mathbf{I} \times \mathbf{I} \times X) \sqcup(\mathbf{I} \times Y)}{\sim}
$$

where $\sim$ is the identification $(t, 1, x)=(t, f(x))$ for $t \in \mathbf{I}$, and $x \in X$. Since $H \circ q$, where $q$ is the quotient map $q:(\mathbf{I} \times \mathbf{I} \times X) \sqcup(\mathbf{I} \times Y) \rightarrow \frac{(\mathbf{I} \times \mathbf{I} \times X) \sqcup(\mathbf{I} \times Y)}{\sim}$, is smooth when restricted to each component of the coproduct $(\mathbf{I} \times \mathbf{I} \times \tilde{X}) \sqcup(\mathbf{I} \times Y), H$ is smooth on the quotient $\mathbf{I} \times \mathbf{I}_{f}$.

We now verify that $(u, H)$ is a representation for $\left(\mathbf{I}_{f}, X\right)$ as an SNDR pair.

- $u^{-1}(0)=\{[0, x]: x \in X\}=i_{0}(X)$

- $H(0,[t, x])=[t, x]$, for all $t \in I$ and $x \in X$, and $H(0,[y])=[y]$, for all $y \in Y$

- $H(s,[0, x])=[0, x]$, for all $s \in I$ and $x \in X$

- If $u([t, x])<1$, then $t<\frac{3}{4}$ and so $\alpha_{2}(t)=0$. Thus, $H(1,[t, x])=[0, x]$.

It is easy to show that $H$ has the FCIP property. This completes the proof.

Finally, we have the following important corollary.

Corollary 5.2. Given any smooth map $f: X \rightarrow Y$, the inclusion $X \hookrightarrow \mathbf{I}_{f}$ is a cofibration with the FCIP. 
Homology, Homotopy and Applications, vol. 9(2), 2007

\section{Baues's first two cofibration axioms}

The classes of morphisms in a cofibration category are the weak equivalences and the cofibrations. A map which is both a cofibration and a weak equivalence is also called a trivial cofibration; see [1] for details.

Lemma 6.1 (Composition Axiom).

1. The composition of two cofibrations is again a cofibration.

2. The product of a cofibration with an identity map is again a cofibration.

Proof.

1. Let $X>^{f}>Y>\stackrel{h}{\longrightarrow} W$ be two cofibrations, $Z$ an arbitrary Frölicher space, and let $k: W \rightarrow Z$ and $K: I \times X \rightarrow Z$ be smooth maps such that $K(0,-)=k \circ h \circ f$. Since $f$ is a cofibration, there is a smooth map $F: I \times$ $Y \rightarrow Z$ such that $F(0,-)=k \circ h$ and $F(t, f(x))=K^{\prime}(t, x)$. Also, since $h$ is a cofibration and $F(0,-)=k \circ h$, we get a smooth map $H: I \times W \rightarrow Z$ such that $H(0,-)=k$ and $H(t, h(y))=F^{\prime}(t, y)$ for all $t \in I$ and $y \in Y$. By easy verification, one has

$$
\begin{aligned}
H(t, h f(x)) & =F^{\prime}(t, f(x)) \\
& =K\left(\left(\alpha_{\mu} \circ \alpha_{\epsilon}\right)(t), x\right) .
\end{aligned}
$$

2. Let $A \stackrel{i}{\longrightarrow} X$ be a cofibration, and let $Y$ and $Z$ be arbitrary Frölicher spaces. Let $G: I \times A \times Y \rightarrow Z$ and $f: X \times Y \rightarrow Z$ be smooth maps such that $f \circ\left(i \times 1_{Y}\right)(a, y)=G(0,(a, y))$ for all $(a, y) \in A \times Y$. By cartesian closedness, we have the following commutative diagram:

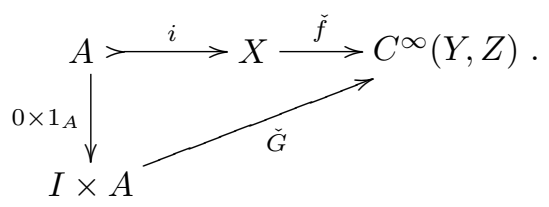

Applying the cofibration property to the map $i$ in the above diagram, we get the following commutative diagram:

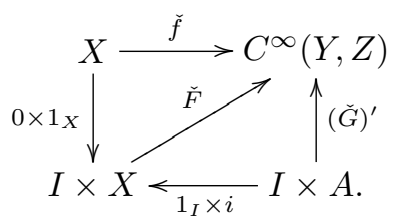

Using cartesian closedness again and Lemma 1.4, one shows that there exists a mapping $F: I \times X \times Y \rightarrow Z$ such that $F(0,(x, y))=f(x, y)$ for all $(x, y) \in$ $X \times Y$, and $F(t,(i(a), y))=G^{\prime}(t,(a, y))$, as required.

We now show that the Pushout Axiom (see [1] ) is also satisfied in the category $\mathbb{F} \mathbb{R} L$ 
Homology, Homotopy and Applications, vol. 9(2), 2007

Lemma 6.2 (Pushout Axiom). For a cofibration $A \stackrel{i}{\longrightarrow} X$, and a map $f: A \rightarrow$ $Y$, there exists the pushout

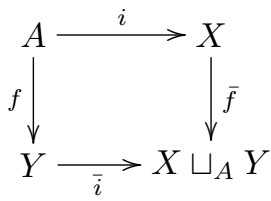

in $\mathbb{F R L}$, with $\bar{i}$ a cofibration. Moreover:

(a) If $f$ is a weak equivalence, so is $\bar{f}$.

(b) If $i$ is a weak equivalence, so is $\bar{i}$.

Proof. We define the adjunction space as usual, i.e. $X \sqcup_{A} Y:=(X \sqcup Y) / \sim$, where $\sim$ is the identification defined by setting $i(a) \sim f(a)$, for $a \in A$. Next, we define smooth maps

$$
\bar{f}: X \rightarrow X \sqcup_{A} Y, x \mapsto[x], x \in X,
$$

and

$$
\bar{i}: Y \rightarrow X \sqcup_{A} Y, y \mapsto[y], y \in Y,
$$

where $[x], x \in X$, and $[y], y \in Y$, denote equivalence classes under the above identification.

To show that $\bar{i}$ is a smooth cofibration, suppose that we have a commutative diagram

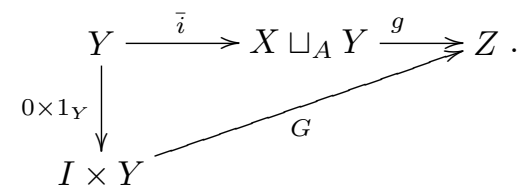

We wish to construct a commutative diagram as follows,

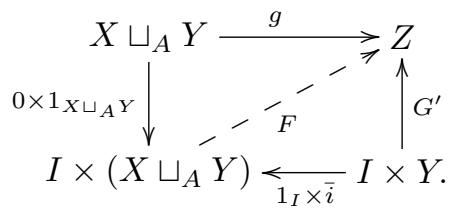

Since $i$ is a cofibration and $g \circ \bar{f} \circ i=\widetilde{G} \circ\left(0 \times 1_{A}\right)$, where $\widetilde{G}=G \circ\left(1_{I} \times f\right)$, there exists a smooth map $H: I \times X \rightarrow Z$, such that $H \circ\left(0 \times 1_{X}\right)=g \circ \bar{f}$, and $H \circ\left(1_{I} \times i\right)=(\widetilde{G})^{\prime}$. Let $F: I \times\left(X \sqcup_{A} Y\right) \rightarrow Z$ be a smooth map defined by setting

$$
F(t, \bar{f}(x))=H(t, x), x \in X
$$

and

$$
F(t, \bar{i}(y))=G^{\prime}(t, y), y \in Y
$$


It is easy to check that $F$ is well defined. Next, we verify that diagram (1) commutes:

$$
F(0, \bar{f}(x))=H(0, x)=g \bar{f}(x), x \in X
$$

and

$$
F(0, \bar{i}(y))=G(0, y)=g \bar{i}(y) .
$$

Thus, the left triangle of (1) commutes. The right triangle of (1) commutes by definition of $F$. Finally, since cartesian closedness of $\mathbb{F} \mathbb{R} \mathbb{L}$ implies that the functor $I \times-$ commutes with colimits, therefore

$$
I \times\left(X \sqcup_{A} Y\right) \simeq(I \times X) \sqcup_{I \times A}(I \times Y) .
$$

Again, since construct products commute with quotients in a cartesian closed topological category, it follows that $1_{I} \times \bar{f}: I \times X \rightarrow(I \times X) \sqcup_{I \times A}(I \times Y)$, and $1_{I} \times$ $\bar{i}: I \times Y \rightarrow(I \times X) \sqcup_{I \times A}(I \times Y)$ are quotients. Since $F \circ\left(1_{I} \times \bar{f}\right)=H$, and $F \circ$ $\left(1_{I} \times \bar{i}\right)=G^{\prime}$ are smooth, $F$ is smooth.

Finally, we need to show that if $i$ is a weak equivalence, then so is $\bar{i}$. We prove this via the following sequence of lemmas.

Lemma 6.3. If $A>^{i}>X$ is a trivial cofibration, then $A$ is a smooth deformation retract of $X$.

Proof. Let $j: X \rightarrow A$ be a homotopy inverse to $i$, and let $F: I \times A \rightarrow A$ be the homotopy giving $j i \simeq_{F} 1_{A}$. We have the commutative diagram

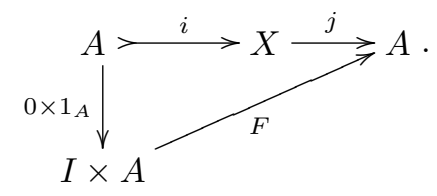

Since $i$ is a cofibration, there exists a smooth map $G: I \times X \rightarrow A$ such that $G(0, x)$ $=j(x)$, for all $x \in X$, and $G(t, i(a))=F^{\prime}(t, a)$, for all $t \in I$ and $a \in A$. Define $k:=$ $G(1,-): X \rightarrow A$. It easily follows that $i k \simeq i j \simeq 1_{X}$; the map $i \circ G$ is the smooth homotopy giving $i k \simeq i j$.

Remark 6.4. The map $k: X \rightarrow A$ in the proof above is such that $k \circ i=1_{A}$, that is, $k$ is a smooth retraction. Again, according to the above proof, the map $G$ is a smooth homotopy giving $j \simeq_{G} k$.

Lemma 6.5. If $A \stackrel{i}{\longrightarrow} X$ is a trivial cofibration with the FCIP and $A$ is closed in the underlying topology of $X$, then $A$ is a strong deformation retract of $X$.

Proof. Let $j: X \rightarrow A$ be a smooth homotopy inverse of $i$, and let $F: I \times A \rightarrow A$ and $G: I \times X \rightarrow X$ be smooth homotopies giving $1_{A} \simeq_{F} j i$ and $1_{X} \simeq_{G} i j$, respectively. By virtue of Remark 6.4, $j$ is homotopic to a retraction; so, we might as well assume from the start that $j$ is a retraction.

Let $P=\mathbf{I} \times X$ and $Q=(\{0,1\} \times X) \cup(\mathbf{I} \times A)$. Since $\{0,1\} \hookrightarrow \mathbf{I}$ and $A \hookrightarrow X$ are SNDR pairs (see Lemma 3.8 and Theorem 4.3 , respectively), so is, by virtue of Theorem 3.9, their product pair $Q \hookrightarrow P$. Define $H_{*}:(0 \times P) \cup(\mathbf{I} \times Q) \rightarrow X$ by 
- $H_{*}(s, 0, x)=x, x \in X, s \in I$

- $H_{*}(s, 1, x)=G\left(\alpha_{\epsilon}(1-s), x\right), x \in X, s \in I$

- $H_{*}(s, t, i(a))=G\left(\alpha_{\epsilon}(1-s) \alpha_{\epsilon}(t), i(a)\right), a \in A, s, t \in I$

- $H_{*}(0, t, x)=G\left(\alpha_{\epsilon}(t), x\right), x \in X, t \in I$.

A routine verification shows that $H_{*}$ is well defined, and the smooth braking function $\alpha_{\epsilon}$ ensures smoothness across the overlaps in the definition of $H_{*}$.

Since $Q \subseteq P$ is closed and $(P, Q)$ an SNDR pair, the inclusion $j: Q \hookrightarrow P$ is a cofibration with the FCIP. Thus, pictorially, we have the following diagram

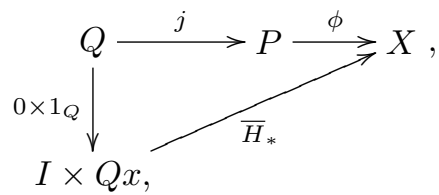

where $\bar{H}_{*}=\left.H_{*}\right|_{\mathbf{I} \times Q}$, and $\phi$ is obtained by solving the equation $H_{*}(0, t, j(x))=\phi \circ$ $j(t, x)$, with $x \in X$. Since $j$ is a cofibration, $\bar{H}_{*}$ admits an extension $H: \mathbf{I} \times P \rightarrow X$. The end value of $H$ at $s=1$ is the required strong retracting deformation.

Corollary 6.6. If $A>\stackrel{i}{\longrightarrow} X$ is a trivial cofibration and $A$ a closed subspace of $X$, then the subspace $A \subseteq \mathbf{I}_{i}$ of the mapping cylinder $\mathbf{I}_{i}$ is a strong deformation retract of $\mathbf{I}_{i}$.

Proof. By Corollary 5.2, the pair $\left(\mathbf{I}_{i}, A\right)$ is an SNDR pair, and hence the inclusion $h: A \hookrightarrow \mathbf{I}_{i}$ is a cofibartion with the FCIP property. We also have the diagram

$$
A \stackrel{h}{\underset{r}{\longrightarrow}} \underset{\mathbf{I}_{i}}{\stackrel{p}{\longrightarrow}} X,
$$

where $r$ is the retraction given by Remark 6.4, and $p$ is given by $p([x])=x$, for all $x \in X$, and $p([t, a])=i(a)$, for all $t \in I$ and $a \in A$. Next, let $q: X \rightarrow \mathbf{I}_{i}$ be the quotient map $x \mapsto[x]$. It is clear that $p q=1_{X}$. The smooth map $H: I \times \mathbf{I}_{i} \rightarrow \mathbf{I}_{i}$, given by

$$
\begin{cases}H(s,[t, a])=[\alpha(s)+(1-\alpha(s)) t, a] & s, t \in I \text { and } a \in A \\ H(s,[x])=[x] & s, t \in I \text { and } x \in X,\end{cases}
$$

where $\alpha: \mathbf{I} \rightarrow \mathbb{R}$ is the braking function such that: $\alpha(t)=0$ if $0 \leqslant t \leqslant \frac{1}{4}, \alpha(t)=1$ if $\frac{3}{4} \leqslant t \leqslant 1,0<\alpha(t)<1$, otherwise, is clearly the smooth homotopy giving $q p \simeq_{H}$ $1_{\mathbf{I}_{i}}$. Therefore, $p$ is a weak equivalence. Since $p$ and $r$ are weak equivalences, so is $h$, by Lemma 6.1. It follows that $h$ is a trivial cofibration with the FCIP property, and one applies Lemma 6.5 to complete the proof.

Remark 6.7. Before we proceed, let us examine the construction of the strong retracting deformation $H(1,-,-): \mathbf{I} \times \mathbf{I}_{i} \rightarrow \mathbf{I}_{i}$, given by Lemma 6.5. Let $j: \mathbf{I}_{i} \rightarrow A$ be given by $j=r p$, where $p$ and $r$ are maps defined in the proof of Corollary 6.6. Using the definitions in the proof of Lemma 6.5, one shows that $H(1,1[1, a])=h(a)$. 
Lemma 6.8. Given the pushout diagram

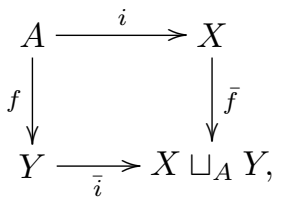

if $A$ is a strong deformation retract of $X$, then $\bar{i}$ is a weak equivalence.

Proof. Let $F: I \times X \rightarrow X$ be the strong retracting deformation such that $F(0, x)$ $=x$, and $F(1, x) \in i(A)$. Define a map $k: X \rightarrow A$ by $k(x)=i^{-1} F(1, x)$. This is smooth since $i$ is an isomorphism of $A$ onto $i(A)$. Define $\bar{j}: X \sqcup_{A} Y \rightarrow Y$ by $\bar{j}([x])=$ $(f \circ k)(x)$, for all $x \in X$, and $\bar{j}([y])=y$, for all $y \in Y$. To see that $\bar{j}$ is well defined on the adjunction, notice that $\bar{j}([i(a)])=f k i(a)=f(a)=\bar{j}([f(a)])$. Finally, we need to verify that $\bar{j}$ is indeed a homotopy inverse to $\bar{i}$. We have $\bar{j} \bar{i}(y)=\bar{j}([y])=y$. On the other hand, $\overline{i j}([y])=\bar{i}(y)=[y]$. Also, notice that $1_{X} \simeq_{F^{-1}} i k=i i^{-1} F(1,-)$, giving us $\left.\bar{i} \bar{j}\right|_{X}=\bar{i} f k=\bar{f} i k \simeq \bar{f}$. That is, $\bar{i} \bar{j}([x]) \simeq[x]$ for $[x] \in X \sqcup_{A} Y$. Notice that this smooth homotopy is well-defined on the adjunction because $F$ is a strong retracting deformation.

Theorem 6.9. Given the pushout diagram

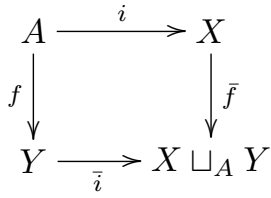

where $i$ is a cofibration, if $i$ is a weak equivalence, then so is $\bar{i}$.

Proof. This proof deals with three different quotient spaces, namely the adjunction space $X \sqcup_{A} Y$, the mapping cylinder of $i, \mathbf{I}_{i}$, and the double mapping cylinder of $i$ and $f, \mathbf{I}_{i, f}$. To make the proof easier to follow, we denote the elements of these spaces as follows. A point in the adjunction $X \sqcup_{A} Y$ is denoted by $[x]$ or $[y]$, where $[i(a)]=[f(a)]$. A point in the mapping cylinder $\mathbf{I}_{i}$ is denoted by $\langle x\rangle$ or $\langle t, a\rangle$, where $\langle 1, a\rangle=\langle i(a)\rangle$. A point in the double mapping cylinder $\mathbf{I}_{i, f}$ is denoted by $\lceil x\rceil$ or $\lceil t, a\rceil$ or $\lceil a\rceil$, where we have $\lceil i(a)\rceil=\lceil 1, a\rceil$ and $\lceil f(a)\rceil=\lceil 0, a\rceil$.

For convenience, we shall sometimes abuse this notation in the following way. For example, suppose that $u \in \mathbf{I}_{i}$. We shall sometimes say that $\lceil u\rceil \in \mathbf{I}_{i, f}$ when we really mean that $u=\langle t, a\rangle$ or $u=\langle x\rangle$ and that $\lceil t, a\rceil$ or $\lceil x\rceil$, respectively, is an element in $\mathbf{I}_{i, f}$. We abuse notation with regard to the adjunction space in a similar way.

Consider the inclusion $h: A \hookrightarrow \mathbf{I}_{i}$. Since $i$ is a trivial cofibration, it follows from Corollary 6.6 that $h(A)$ is a strong deformation retract of $\mathbf{I}_{i}$. We then have a homotopy $F: I \times \mathbf{I}_{i} \rightarrow \mathbf{I}_{i}(\operatorname{rel} h(A))$, with the properties that $F(0,\langle x\rangle)=\langle x\rangle$, and $F(1,\langle x\rangle) \in h(A)$, for all $\langle x\rangle \in \mathbf{I}_{i}$. Remark 6.7 shows that we may choose $F$ so that 
$F(1,\langle i(a)\rangle)=F(1,\langle 1, a\rangle)=h(a)$. We have the following diagram

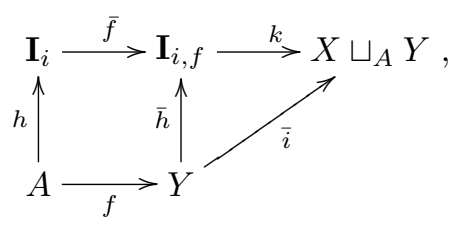

where $\bar{h}$ is the weak equivalence given by Lemma 6.8 and $k$ is defined below. We shall show that $k$ is a weak equivalence, and that the right triangle is commutative with our definition of $k$. This will imply that $\bar{i}$ is a weak equivalence.

Define $P: I \times \mathbf{I}_{i, f} \rightarrow \mathbf{I}_{i, f}$ by

- $P(s,\lceil x\rceil)=\lceil F(s,\langle x\rangle)\rceil$

- $P(s,\lceil t, a\rceil)=\lceil F(s,\langle t, a\rangle)\rceil$

- $P(s,\lceil y\rceil)=\lceil y\rceil$.

$P$ is well defined, since $P(s,\lceil i(a)\rceil)=\lceil F(s,\langle i(a)\rangle)\rceil=\lceil F(s,\langle 1, a\rangle)\rceil$. Also,

$$
P(s,\lceil 0, a\rceil)=\lceil F(s,\langle 0, a\rangle)\rceil=\lceil 0, a\rceil=\lceil f(a)\rceil .
$$

Now, define $\bar{k}: X \sqcup_{A} Y \rightarrow \mathbf{I}_{i, f}$ by $\bar{k}([x])=\lceil F(1,\langle x\rangle)\rceil$, and $\bar{k}([y])=\lceil y\rceil$. Notice that $\bar{k}$ is well defined. Next, define $k: \mathbf{I}_{i, f} \rightarrow X \sqcup_{A} Y$ by $k(u)=[P(1, u)]$, for $u \in \mathbf{I}_{i, f}$. We now observe that $\bar{k}$ is a homotopy inverse to $k:=[P(1,-)]$. It is clear that $P(0,-)=1_{\mathbf{I}_{i, f}}$. Notice that

$$
P(1,\lceil x\rceil)=\lceil F(1,\langle a\rangle)\rceil,
$$

where $F(1,\langle x\rangle) \in \bar{f}(\bar{h}(A)) \subset \mathbf{I}_{i, f}$, and hence $\bar{k} k(\lceil x\rceil)=k(\lceil x\rceil)=P(1,\lceil x\rceil)$. Likewise $P(1,\lceil t, a\rceil)=\lceil F(1,\langle t, a\rangle)\rceil$, and so $\bar{k} k(\lceil t, a\rceil)=P(1,\lceil t, a\rceil)$, and $P(1,\lceil y\rceil)=\lceil y\rceil=$ $\bar{k} k(\lceil y\rceil)$. Thus, $1_{\mathbf{I}_{i, f}} \simeq_{P} \bar{k} k$.

For the other direction, first define $q: \mathbf{I}_{i, f} \rightarrow X \sqcup_{A} Y$ by

- $q(\lceil x\rceil)=[x]$

- $q(\lceil t, a\rceil)=[f(a)]=[i(a)]$

- $q(\lceil y\rceil)=[y]$.

Clearly, $q$ is well defined. Notice that we have $[x]=q P(0,\lceil x\rceil)$, and $[y]=q P(0,\lceil y\rceil)$. Thus, $1_{X \sqcup_{A} Y}=q P(0,\lceil-\rceil)$. At the other endpoint, we have

$$
q P(1,\lceil x\rceil)=[P(1,\lceil x\rceil)]=k \bar{k}([x]),
$$

and

$$
q P(1,\lceil y\rceil)=[P(1,\lceil y\rceil)]=k(\lceil y\rceil)=k \bar{k}([y]) .
$$

Thus, $1_{X \sqcup_{A} Y} \simeq_{q P} k \bar{k}$. Clearly, $k \circ \bar{h}=\bar{i}$.

We have shown that $k$ is a weak equivalence. Since $\bar{h}$ is a weak equivalence, we deduce by the Composition Axiom (cf. Lemma 6.1) that $\bar{i}$ is a weak equivalence. 
Homology, Homotopy and Applications, vol. 9(2), 2007

\section{The exact sequence of a cofibration}

Our aim in this section is to show how one can use SNDR pairs to prove the existence of the right exact Puppe sequence. We state the result in Theorem 7.1 and break the proof of the result up into a number of lemmas. We follow the method used by Whitehead [13] for the topological case.

Throughout this section we work in the category $\mathbb{F R L}_{*}$ of pointed Frölicher spaces, and basepoint preserving smooth maps.

Theorem 7.1. Let $W$ be an object in $\mathbb{F} \mathbb{R} \mathbb{L}_{*}$, and suppose that $i: A \hookrightarrow X$ is a cofibration in $\mathbb{F} \mathbb{R L}_{*}$. For any basepoint $x_{0} \in A \subseteq X$ there is a sequence

$$
\begin{aligned}
& \cdots \longrightarrow\left[\sum^{n+1} A, W\right] \stackrel{\left(\sum^{n} k\right)^{*}}{\longrightarrow}\left[\sum^{n} \mathbf{T}_{i}, W\right] \stackrel{\left(\sum^{n} j\right)^{*}}{\longrightarrow}\left[\sum^{n} X, W\right] \stackrel{\left(\sum^{n} i\right)^{*}}{\longrightarrow}\left[\sum^{n} A, W\right] \longrightarrow \cdots \\
& \cdots \longrightarrow\left[\sum A, W\right] \stackrel{k^{*}}{\longrightarrow}\left[\mathbf{T}_{i}, W\right] \stackrel{j^{*}}{\longrightarrow}[X, W] \stackrel{i^{*}}{\longrightarrow}[A, W]
\end{aligned}
$$

which is an exact sequence in $\mathbb{S E T S}_{*}$, where $j: X \rightarrow \mathbf{T}_{i}$ is the inclusion discussed in Paragraph 2.3 and $k: \mathbf{T}_{i} \rightarrow \sum A$ is the quotient map defined below.

It is, in fact, possible to prove that the sequence above is an exact sequence of groups as far as $\left[\sum A, W\right]$ and that the morphisms to this point are group homomorphisms, but we shall not do so here.

The reduced (flattened) suspension of a pointed Frölicher space $X$ is defined as

$$
\sum X=(\mathbf{I} /\{0,1\}) \wedge X,
$$

where the reduced join is defined as for topological spaces with the identified set taken as basepoint, and with 0 the basepoint of $\mathbf{I}$.

In this section, whenever we refer to the suspension of a space, we mean the reduced flattened suspension defined above.

Lemma 7.2. If $(x, A)$ is an SNDR pair and $p: X \rightarrow X / A$ the quotient map, then the sequence

$$
A \stackrel{i}{\longrightarrow} X \stackrel{p}{\longrightarrow} X / A
$$

is right exact.

Proof. To show that the given sequence is right exact we must show that for any Frölicher space $W$ the following sequence is exact in $\mathbb{S E T S}$ :

$$
[X / A, W] \stackrel{p^{*}}{\longrightarrow}[X, W] \stackrel{i^{*}}{\longrightarrow}[A, W] .
$$

It is easy to see that im $p^{*} \subseteq$ ker $i^{*}$. To see the reverse inclusion, let $g: X \rightarrow W$ be an element of $[X, W]$, with $\left.g\right|_{A} \simeq w_{0}\left(\right.$ rel $\left.w_{0}\right)$, where $w_{0} \in W$. Since $A \stackrel{i}{\longrightarrow} X$ is an SNDR pair, the map $i$ is a cofibration, and so we may extend $w_{0}$ to a smooth map $g^{\prime}: X \rightarrow W$ such that $g^{\prime} \simeq g$. But $g^{\prime}$ is constant on $A$, and so there exists a smooth map $g_{1}: X / A \rightarrow W$ such that $p^{*}\left(g_{1}\right)=g^{\prime}$. This shows that ker $i^{*} \subset \operatorname{im} p^{*}$. 
Homology, Homotopy and Applications, vol. 9(2), 2007

Lemma 7.3. For any smooth map $f: X \rightarrow Y$, the sequence

$$
X \stackrel{f}{\longrightarrow} Y \stackrel{l}{\longrightarrow} \mathbf{T}_{f}
$$

is right exact, where $l$ is the usual inclusion of $Y$ into the mapping cone; i.e. $y \mapsto$ $[y] \in \mathbf{T}_{f}$.

Proof. One can show that there is a homotopy commutative diagram

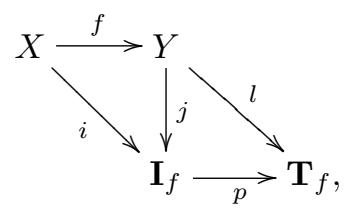

where $i, j$, and $l$ are the usual inclusions, and $p$ is the quotient map that collapses away $\{0\} \times X$ to a point. Since, by Theorem $5.1,\left(\mathbf{I}_{f}, X\right)$ is an SNDR pair, it follows from Lemma 7.2 that the sequence

$$
X \stackrel{i}{\longrightarrow} \mathbf{I}_{f} \stackrel{p}{\longrightarrow} \mathbf{T}_{f}
$$

is right exact. It is fairly easy to show that $j: Y \rightarrow \mathbf{I}_{f}$ is a homotopy equivalence. Therefore, the sequence

$$
X \stackrel{f}{\longrightarrow} Y \stackrel{l}{\longrightarrow} \mathbf{T}_{f}
$$

is right exact.

Lemma 7.4. For any smooth map $i: A \rightarrow X$, there is an infinite right exact sequence

$$
A \stackrel{i}{\longrightarrow} X \stackrel{i^{1}}{\longrightarrow} \mathbf{T}_{i} \stackrel{i^{2}}{\longrightarrow} \cdots \stackrel{i^{n-1}}{\longrightarrow} \mathbf{T}_{i^{n-2}} \stackrel{i^{n}}{\longrightarrow} \mathbf{T}_{i^{n-1}} \stackrel{i^{n+1}}{\longrightarrow} \cdots,
$$

where $i^{n}, n \geqslant 1$, are inclusion maps.

Proof. The pair $\left(\mathbf{T}_{i}, X\right)$ is an SNDR pair. The representation for the pair $\left(\mathbf{I}_{f}, X\right)$ in Theorem 5.1 can be adapted to show this. One iterates the procedure of Lemmas 7.2 and 7.3.

One can easily see that there is an isomorphism between $\mathbf{T}_{i} / X$ and $\sum A$. Define $q: \mathbf{T}_{i} \rightarrow \sum A$ to be the map which identifies $X \subset \mathbf{T}_{i}$ to a point, followed by the isomorphism $\mathbf{T}_{i} / X \rightarrow \sum A$.

Lemma 7.5. The sequence

$$
X \stackrel{i^{1}}{\longrightarrow} \mathbf{T}_{i} \stackrel{q}{\longrightarrow} \sum A
$$

is right exact. 
Proof. As noted above the pair $\left(\mathbf{T}_{i}, X\right)$ is an SNDR pair. We have the commutative diagram

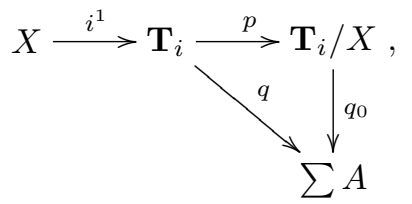

where $p: \mathbf{T}_{i} \rightarrow \mathbf{T}_{i} / X$ is the identification map, and $q_{0}: \mathbf{T}_{i} / X \rightarrow \sum A$ is an isomorphism. The top line of the diagram is right exact, by Lemma 7.2, and so the sequence

$$
X \stackrel{i^{1}}{\longrightarrow} \mathbf{T}_{i} \stackrel{q}{\longrightarrow} \sum A
$$

is right exact.

There is a commutative diagram

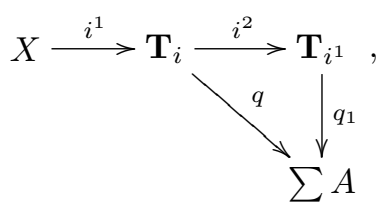

where $q_{1}$ is a homotopy equivalence. (See Whitehead [13] for more details of this map.) Using commutative diagrams of this form, one can now proceed almost exactly as one does in the topological situation, as in Whitehead [13] for example, to get the following infinite right exact sequence:

$$
\begin{gathered}
A \stackrel{i}{\longrightarrow} X \stackrel{i^{1}}{\longrightarrow} \mathbf{T}_{i} \stackrel{q}{\longrightarrow} \sum A \stackrel{\sum i}{\longrightarrow} \sum X \stackrel{\sum i^{1}}{\longrightarrow} \sum \mathbf{T}_{i} \stackrel{\sum q}{\longrightarrow} \cdots \\
\cdots \longrightarrow \sum^{n} A \stackrel{\sum^{n} i}{\longrightarrow} \sum^{n} X \stackrel{\sum^{n} i^{1}}{\longrightarrow} \cdots .
\end{gathered}
$$

The definition of right exactness now gives us the exact sequence of Theorem 7.1.

\section{References}

[1] H. J. Baues, Algebraic Homotopy, Cambridge University Press, Cambridge, 1989.

[2] A. Cap, $K$-theory for convenient algebra, Dissertationen, Faculty of Mathematics, University of Vienna, 1993.

[3] P. Cherenack, Applications of Frölicher spaces to cosmology, Ann. Univ. Sci. Budapest 41 (1998), 63-91.

[4] P. Cherenack, Frölicher versus differential spaces: a prelude to cosmology, Papers in Honour of Bernhard Banaschewski (Cape Town, 1996), 391-413, Kluwer Academic Publishers, Dordrecht (2000). 
[5] P. Cherenack, The left exactness of the smooth left Puppe sequence, New Developments in Differential Geometry (Debrecen, 1994), 59-76, Mathematics and Its Applications, 350, Kluwer Academic Publishers, Dordrecht, 1996.

[6] P. Cherenack, Smooth homotopy, Topology with applications, (Szekszárd, 1993), 47-70, Bolyai Society Mathematical Studies, 4, János Bolya Mathematical Society, Budapest, 1995.

[7] B. Dugmore, The right exactness of the smooth right Puppe sequence, Master's Thesis, University of Cape Town, 1996.

[8] A. Frölicher and A. Kriegl, Linear Spaces and Differentiation Theory, J. Wiley and Sons, Chichester, 1988.

[9] M. W. Hirsch, Differential Topology, Graduate Texts in Math. 33, SpringerVerlag, New York, 1976.

[10] I. M. James, General Topology and Homotopy Theory, Springer-Verlag, New York, 1984.

[11] A. Kriegl and P. Michor, The Convenient Setting of Global Analysis, Mathematical Surveys and Monographs, 53, American Mathematical Society, Providence, RI, 1997.

[12] J. Nestruev, Smooth Manifolds and Observables, Springer-Verlag New York, 2003.

[13] G. W. Whitehead, Elements of Homotopy Theory, Springer-Verlag, New York, 1978.

Brett Dugmore brettmo@iafrica.com

Cadiz Securities

Newlands 7700

Cape Town, South Africa

Patrice Pungu Ntumba patrice.ntumba@up.ac.za

Department of Mathematics and Applied Mathematics

University of Pretoria

Hatfield 0002, South Africa

This article is available at http://intlpress.com/HHA/v9/n2/a15 